\title{
Social Cognitive Interventions in Neuropsychiatric Patients: A Meta-Analysis
}

\author{
Renée L. Roelofs, ${ }^{1,2,3}$ Ellen Wingbermühle,, ${ }^{1,2}$ Jos I.M. Egger ${ }^{1,2,3,4}$ \\ and Roy P.C. Kessels ${ }^{2,4,5}$ \\ ${ }^{1}$ Centre of Excellence for Neuropsychiatry, Vincent van Gogh Institute for Psychiatry, Venray, the \\ Netherlands \\ ${ }^{2}$ Donders Institute for Brain, Cognition and Behaviour, Radboud University, Nijmegen, the Netherlands \\ ${ }^{3}$ Behavioural Science Institute, Radboud University, Nijmegen, the Netherlands \\ ${ }^{4}$ Centre of Excellence for Korsakoff and Alcohol-Related Cognitive Disorders, Vincent van Gogh Institute \\ for Psychiatry, Venray, the Netherlands \\ ${ }^{5}$ Department of Medical Psychology, Radboud University Medical Center, Nijmegen, the Netherlands
}

\begin{abstract}
Social cognitive deficits are common in neuropsychiatric disorders. Given the proximity of social cognition (SC) to everyday functioning, many intervention studies (including targeted, comprehensive, and broad-based approaches) have focussed on SC. The aim of this paper was to quantitatively meta-analyse the efficacy of SC interventions in adult neuropsychiatric patients. Databases Pubmed, PsycINFO, Web of Knowledge, and Embase were searched for controlled SC intervention studies published between 01-01-2003 and 01-01-2016. Forty-one studies, comprising 1,508 patients with schizophrenia, autism spectrum disorders, or acquired brain injury were included. Outcome measures evaluated emotion perception (EP), social perception (SP), Theory of Mind (ToM), and social functioning (SF). The meta-analyses showed that interventions were effective in improving SC (Cohen's $d=.71$ ). Interventions targeting one specific SC function were found to be most effective $(d=.89)$, followed by broad-based interventions, targeting non-SC domains in addition to $S C(d=.65)$, and comprehensive interventions, that target multiple SC processes $(d=.61)$. Targeted interventions were especially effective in improving EP and ToM. Comprehensive interventions were able to ameliorate $\mathrm{EP}$, ToM, and SF. Broad-based interventions were especially effective in improving SF, but also showed effects on EP and ToM.
\end{abstract}

Keywords: Social cognition, training, treatment, psychotherapy, neuropsychiatry, emotion perception, social perception, theory of mind, social functioning, systematic review

\section{Introduction}

Social cognitive deficits are common in a variety of psychiatric and neurological disorders. Given the proximity of social cognition (SC) to everyday functioning, many intervention studies have focussed on improving SC in these populations. The aim of this paper is to quantitatively meta-analyse the efficacy of these interventions in adult neuropsychiatric patients.

\section{Defining Social Cognition}

SC is considered to be a cognitive domain that includes the mental processes underlying social

Address for correspondence: R.L. Roelofs, Centre of Excellence for Neuropsychiatry, Vincent van Gogh Institute for Psychiatry, Stationsweg 46, 5803 AC Venray, the Netherlands. Phone: +31 478527 339; Telefax: +31 478527626.

E-mail: rroelofs@vvgi.nl 
interactions. In other words, SC represents how people make sense of other people and themselves (Fiske \& Taylor, 2013). SC is commonly conceptualised as three components of information processing (Adolphs, 2001; Beer \& Ochsner, 2006): (1) perception of socially relevant information (of the self, other people, or the interaction between the two), (2) interpretation of this information and the integration with contextual information, and (3) execution and regulation of social behaviour.

In accordance with this definition, three cognitive processes are commonly distinguished in SC intervention studies and also used as outcome measures. These include emotion perception, social perception, and Theory of Mind (ToM) (Green, Olivier, Crawley, Penn, \& Silverstein, 2005; Horan, Kern \& Green, 2008). Emotion perception refers to the ability to perceive emotional information from the self (inner feelings) or others (in facial expressions or voices for instance). Social perception is conceptualised as the ability to identify social cues from contextual information and communicative gestures, such as awareness of social roles, rules, and social relationships. ToM is defined as mental state attribution or mentalising, and it refers to the ability to infer the intentions, beliefs, feelings, and opinions of the self and others. Fourth, social functioning encompasses social behaviours that are the result of the aforementioned cognitive processes, for instance, interpersonal contact or social skills. Alexithymia is a personality concept that is strongly related to SC, which is characterised by an emotion regulation deficit, difficulties in identifying and describing own emotional feelings, a limited imaginal capacity, and an externally oriented cognitive style (Taylor \& Bagby, 2012). A considerable body of evidence suggests that alexithymia is often associated with SC impairments, such as deficits in emotion recognition (Bird \& Cook, 2013; Grynberg et al., 2012; Lane et al., 1996; Lane, Sechrest, Riedel, Shapiro \& Kasniak, 2000; Mann, Wise, Trinidad \& Kohanski, 1995; Taylor, Taylor \& Bagby, 1993) and mentalising (Moriguchi et al., 2006; SubicWrana, Beutel, Knebel \& Lane, 2010; Swart, Kortekaas \& Aleman, 2009).

\section{Social Cognition in Neuropsychiatric Patients}

SC deficits have been demonstrated in a variety of neuropsychiatric disorders (e.g., schizophrenia: Penn, Corrigan, Bentall, Racenstein, \& Newman, 1997; autism spectrum disorders (ASD): Couture et al., 2010; mood disorders, Samamé, 2013; anxiety disorders: Plana, Lavoie, Battaglia \& Achim, 2014; traumatic brain injury: Muller et al., 2010; Alzheimer's dementia: Bediou et al., 2009; frontotemporal dementia: Gregory et al., 2002; Lough et al., 2006; Huntington's disease: Snowden et al., 2003). Furthermore, impaired SC has also been reported in individuals with intellectual disabilities (Baurain \& Nader-Grosbois, 2013; Leffert, Siperstein \& Widaman, 2010) and genetic syndromes (e.g., Williams syndrome: Meyer-Lindenberg et al., 2005; Prader-Willi syndrome: Whittington \& Holland, 2011; Noonan syndrome: Wingbermühle, Egger, Verhoeven, Van der Burgt \& Kessels, 2012; Turner syndrome: Burnett, Reutens \& Wood, 2010; Phelan-McDermid syndrome: Egger, Zwanenburg, Van RavenswaaijArts, Kleefstra \& Verhoeven, 2016).

Poor SC is associated with psychological distress, social isolation, and reduced self-esteem (Beauchamp \& Anderson, 2010). Alexithymia has been shown to diminish quality of life and is associated with difficulty in interpersonal relationships (Kennedy \& Franklin, 2002). It is also thought to be a risk factor for various (psycho)somatic and psychiatric disorders (for instance, mood and anxiety disorders), although only correlational data are available to date, making causal inferences not possible (Taylor \& Bagby, 2012).

In patients with schizophrenia, associations have been found between SC (emotion perception, social perception, and ToM) and functional outcome measures such as community functioning, social behaviour, social problem solving, and social skills (Couture, Penn \& Roberts, 2006). Fett et al. (2011) even found SC to be more strongly related to community functioning than 'cold' neurocognitive processes (i.e., cognitive functions involved in non-affective information processing, such as processing speed, attention, [working] memory, and problem solving). Penn et al., (1997) stated that SC differs from neurocognition in the type of stimuli processed (social, personally relevant, mutable vs. non-social, neutral, stable), the relationship of the observer to the stimulus (multidirectional vs. unidirectional), and the evaluation of the performance. Given the proximity of SC to community functioning (i.e., interpersonal relations, work functioning), SC is also an important treatment target in addition to neurocognitive (dys)function in isolation.

\section{Treatments for Social Cognition}

Regarding the influence of SC on the functional status of patients with schizophrenia and its established responsiveness to treatment, research concerning SC interventions in other psychiatric disorders has increased rapidly over the past decade. Most studies categorise SC treatments into three 
groups: targeted, comprehensive, or broad-based interventions (Fiszdon \& Reddy, 2012). Targeted interventions focus on a single SC subdomain, like emotion perception (e.g., Treatment of Affect Recognition (TAR): Wölwer et al., 2005). Comprehensive interventions also solely focus on $\mathrm{SC}$, but address a broader range of SC subdomains and impairments. For instance, the Social Cognition Interaction Training (SCIT) (Roberts, Penn \& Combs, 2016) encompasses treatment of emotion perception, ToM, and attributional bias. Broad-based interventions embed training of SC aspects within the context of other interventions, such as social skills training or neurocognitive remediation. The Cognitive Enhancement Therapy (CET) is a well-known example of a broadbased intervention, which combines a SC intervention with neurocognitive training (Hogarty \& Flesher, 1999).

By far, the majority of SC interventions have focussed on reducing impairments in patients with schizophrenia spectrum disorders (SSD). Several reviews and one meta-analysis have been published in the field of schizophrenia research (e.g., Fiszdon \& Reddy, 2012; Horan et al., 2008; Kurtz \& Richardson, 2011). These reviews concluded that targeted, comprehensive, and broad-based interventions are all able to enhance relatively 'simple' SC processes (e.g., emotion perception), whereas more complex, higher order functions such as ToM and social perception have proven to be less amenable for treatment (Fiszdon \& Reddy, 2012; Kurtz \& Richardson, 2011). Especially targeted interventions improve specific SC functions in SSD, whilst improvement in multiple domains has been reported for comprehensive and broadbased interventions (Choi, Kim, Lee \& Green, 2009; Horan et al., 2008; Kurtz \& Richardson (2011); Mueller, Schmidt \& Roder, 2013; Statucka $\&$ Walder 2013). The common multi-component design of the broad-based and comprehensive interventions complicates the assignment of specific intervention effects and the indication of key ingredients (Choi et al., 2009; Horan et al., 2008; Roberts \& Velligan, 2012; Roberts et al., 2014). The high degree of similarity between the training stimuli and test stimuli in targeted interventions has raised concern about 'training to the task' and the (lack of) transfer to real-life social functioning has appeared to be problematic (Horan et al., 2008; Paquin, Wilson, Cellard, Lecomte \& Potvin, 2014; Wölwer \& Frommann, 2011). In addition, Peyroux and Franck (2014) stated that SC interventions rely too heavily on hierarchical, step-bystep models, which train one component at a time, lacking the complex demands of real-life social interactions.
Interventions addressing SC in ASD or in patients with acquired brain injury (ABI) have been studied less frequently than those in SSD. In patients with ABI, SC interventions mainly focussed on retraining social cue perception, social communication, and social skills or functioning. Although the number of studies so far is limited, there are positive indications from previous literature reviews that emotion perception, social communication skills, and social functioning can be improved after brain injury (Braden, 2014; Driscoll, Dal Monte \& Grafman, 2011; Manly \& Murphy, 2012). A systematic review on psychosocial interventions (including SC interventions) in adults with ASD reported that these treatments are generally effective, although the quantity and quality of the studies are again limited (Bishop-Fitzpatrick, Minshew \& Eack, 2013). A meta-analysis on targeted interventions of ToM and precursor skills (such as emotion perception) in children and adults with ASD showed modest evidence of treatment success, but studies lacked generalisation to novel contexts and the long-term maintenance of learnt skills was unclear (Fletcher-Watson, McConnell, Manola \& McConachie, 2014).

Several studies focussed on alexithymia in psychotherapeutic treatments, but only a few interventions have been described that were specifically designed to remediate alexithymic problems. Alexithymia is considered to be a difficult construct to treat, because most interventions require some awareness of and access to the patient's own emotions, skills that are commonly impaired in alexithymic individuals (Byrne, Bogue, Egan, \& Lonergan, 2014; Ogrodniczuk, Sochting, Piper \& Joyce, 2012). Cameron, Ogrodniczuk, and Hadjipavlou (2014) reviewed 23 studies that examined changes in alexithymia levels, following a psychological intervention. Patient populations included eating disorders $(k=4)$, anxiety disorders $(k=4)$, mood disorders $(k=1)$, substance use disorders $(k=2)$, mixed in- and outpatient populations $(k=5)$, non-psychiatric medical conditions $(k=5)$, and non-patient populations $(k=2)$. The authors concluded that especially the studies that targeted alexithymia directly tended to report significant reductions in alexithymia levels, whilst psychological interventions that were not primarily intended to treat alexithymia showed more inconsistent results.

\section{Aims of This Meta-analysis}

Since SC impairments are common in a variety of neuropsychiatric disorders (Bediou et al., 2009; Couture et al., 2010; Gregory et al., 2002; Lough et al., 2006; Muller et al., 2010; Penn et al., 1997; 
Plana et al., 2014; Samamé, 2013; Snowden et al., 2003) and are known to affect psychological wellbeing, it is of clinical importance to investigate how SC deficits can be effectively treated in these patient groups. Therefore, the aim of the present paper is to conduct a meta-analysis on all published evidence regarding the effects of SC interventions on SC and alexithymia in adult neuropsychiatric patients, that is, patients with psychiatric diseases and/or neurological conditions, for which evident neuropsychological disorders have been described.

Furthermore, we investigated the effects of the three different treatment types (targeted, comprehensive, and broad-based) on SC and alexithymia, both in the general population of neuropsychiatric patients as well as for specific patient groups. To our knowledge, no systematic reviews are available that investigate SC interventions across different patient groups and treatment types. This metaanalysis will be of relevance to clinical and academic professionals, since it provides information about required methods and intensity of SC interventions, and the applicability to different patient groups. The findings of this systematic review can subsequently be used to develop new, evidencebased interventions for the enhancement of SC in other adult neuropsychiatric patient populations.

\section{Methods}

\section{Inclusion Criteria}

In the current study, SC interventions were defined as psychological, skill-based treatments, designed to ameliorate one of the five $\mathrm{SC}$ aspects, described in more detail in the introduction (i.e., emotion perception, social perception, ToM, social functioning, and alexithymia). At least one SC outcome measure needed to be included at post-treatment assessment. Medical interventions (e.g., oxytocin nasal spray, Guastella \& MacLeod, 2012) and alternative, non-psychological treatments, such as yoga therapy (Bangalore \& Varambally, 2012), and drama therapy (Corbett et al., 2011; Lerner, Mikami \& Levine, 2010) did not meet this definition.

Neuropsychiatric disorders were defined as psychiatric diseases and/or neurological conditions, for which evident neuropsychological problems have been described. Based on this definition, patients with SSD, ASD, dementia, brain injury, or mood and anxiety disorders could be included, whilst for example patients with eating disorders, personality disorders, or etiologically unspecified intellectual disability were excluded. Furthermore, given the influence of cultural differences on (social)cognition (Varnum, Grossman, Kitayama \&
Nisbett, 2010), this study only focussed on patients in Western societies (i.e., Europe - including Turkey, United States, Canada, Australia, and New Zealand). Lastly, studies including patients younger than 18 years or patients with a comorbid diagnosis of substance dependency or abuse were also excluded.

In sum, to be included in this systematic review, studies must have (1) investigated a SC intervention or alexithymia treatment, (2) used at least one behavioural measure of SC as a primary outcome measure at post-treatment assessment, (3) included a sample of adult ( $>18$ years) neuropsychiatric patients, (4) contained a patient control group, (5) used a sample-size of more than one patient per group, (6) investigated patients in Western societies, (7) presented original data (no abstracts, reviews, conference proceedings, research protocols), (8) been published in peer-reviewed journals, and (9) been written in the English language.

\section{Search Methods}

All available published literature from January 1, 2003 up to January 1, 2016 was systematically searched using the electronic databases of PubMed, PsycINFO, Web of Knowledge, and Embase. The following search terms (or combinations/truncated versions) were used: 'social cognition', 'affective information processing', 'training', 'intervention', 'rehabilitation', and 'treatment'. Additional searches were performed, using the more focussed search terms: 'autism', 'theory of mind', 'alexithymia', 'brain injury', or 'brain damage'. Subsequently, the reference lists of the included studies and relevant review articles were scanned for additional eligible studies.

\section{Selection Procedure}

Articles were selected and scrutinised according to the PRISMA guidelines (Preferred Reporting Items of Systematic reviews and Meta-Analyses, Moher, Liberati, Tetzlaff \& Altman, 2009). In Figure 1, the selection procedure is depicted in a flow diagram. All hits were screened on title or abstract by the first author. All citations obviously irrelevant to the topic were directly excluded and duplicates were removed. Subsequently, two of the authors (RLR and EW) independently assessed and selected the remaining studies. Any discrepancies were resolved by discussion between these authors, resulting in consensus.

To avoid bias due to duplicate results, studies that used data or patient samples identical to other included studies were excluded. In such cases, RLR and EW discussed the overlapping studies and came to an agreement about which study 


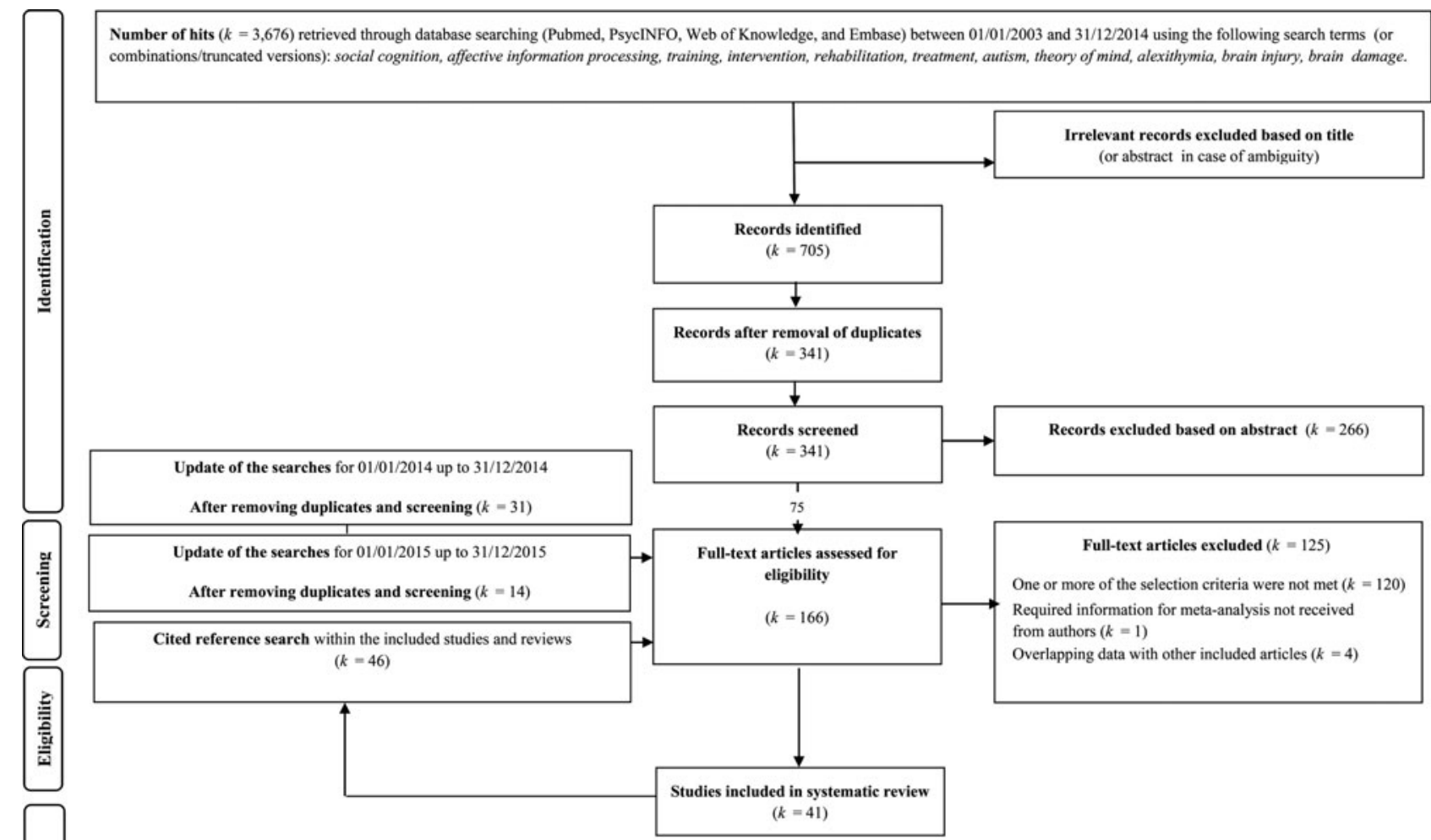

FIGURE 1

PRISMA flow diagram of selection procedure. 
should be excluded. Here, more recent studies and those with larger sample sizes were preferred over older studies or those reporting preliminary data. Furthermore, one study had to be excluded because the requested information for the meta-analysis was not available in a timely fashion.

\section{Data Extraction}

The following data were extracted from the selected studies: population, sample size, design, name and type of intervention, intervention target(s), control group, age of participants, duration and dose of intervention, group size, SC outcome measures, availability of follow-up data, and main findings.

The included intervention studies were divided into three categories: targeted, comprehensive, or broad-based interventions. For the purpose of this review, the following classification was used: Targeted interventions should focus on one SC impairment; comprehensive interventions should focus on two or more SC impairments; and broadbased interventions should focus on one or more SC impairments within other (non-SC) treatment programmes, which themselves could not be classified as targeted or comprehensive.

If studies used more than one control group (e.g., both a wait-list control group and a treatmentas-usual condition), the most frequently used control group was included in the meta-analysis. For studies including more than one SC intervention group, the authors discussed which intervention was included in the meta-analysis. Here, criteria for selection were the focus of the article, the sample size of the two treatment groups, and the additional informative value of the intervention (preference for new interventions over already included interventions).

SC outcome measures were grouped into four domains: emotion perception, social perception, ToM, and social functioning. Measures of social functioning were very diverse, encompassing social behaviour but also non-social activities of daily living. To reduce heterogeneity, in this systematic review only (subscale) scores were included that primarily assessed social behaviour in social interactions. For studies that presented a composite score to reflect SC, only relevant subscores were included. Outcome measures that were specific for the patient group under study (for instance, attributional bias or jumping to conclusions in schizophrenia studies) were not included in the review. For an overview of the included outcome measures, see Table 1. All selected outcome measures had to generate continuous data. Means and standard deviations of post-treatment assessment were selected for the meta-analyses.

\section{Data Analysis}

MetaWin version 2.1 (www.metawinsoft.com) was used to calculate Hedges' g based on posttreatment means (or adjusted means) and standard deviations. If lower scores represented a better performance, scores were multiplied by -1 before being entered into MetaWin. Subsequently, pooled standardised mean differences and variances were calculated per study for a total SC score and for the four SC domains. Positive values indicated improvement. When studies included more than one outcome measure per domain, the standardised mean differences and their variances were averaged to avoid studies with multiple outcome measures having more influence in the analyses. The pooled standardised mean differences and sample sizes were entered into RevMan version 5.3 (www. tech.cochrane.org/revman). The inverse-variance random-effects method was used, since it was assumed that the studies were estimating different, yet related, intervention effects (Deeks, Higgins \& Altman, 2008).

First, the overall effect of the three treatment types (targeted, comprehensive, broad-based) on SC was studied. Subgroup analyses were performed for the effects of treatment type on SC for the different patient populations.

Heterogeneity of intervention effects was assessed by the $\chi^{2}$ test. The $I^{2}$ statistic was used to quantify the inconsistency, reflecting the percentage of the variability in effect estimates that is due to heterogeneity rather than chance (Deeks et al., 2008). A heterogeneity over $50 \%$ was considered substantial. As an estimate of the between-study variance in random-effects meta-analyses, $\tau^{2}$ was presented. The square root of $\tau^{2}$ represents the estimated standard deviation of underlying effects across studies (Deeks et al., 2008).

In order to address a potential publication bias, fail-safe $N$ were calculated using the method of Rosenthal (1979). The PEDro-P scale (available on the website of PsycBITE, www.psycbite.com) was used as a measure of internal validity and methodological quality of the included studies (total score ranging from 0 to 10 ). Studies were scored by two of the authors (RLR and EW) in order to generate consensus ratings. Maher, Sherrington, Herbert, Moseley, and Elkins (2003) indicated that the reliability of the total PEDro score, based on consensus judgements, is acceptable. They concluded that the scale has sufficient reliability for use in systematic reviews. 


\section{TABLE 1}

Included Social Cognitive Outcome Measures for Emotion Perception, Social Perception, Theory of Mind, and Social functioning

\begin{tabular}{|c|c|}
\hline $\begin{array}{l}\text { Social cognitive } \\
\text { domain }\end{array}$ & Social cognitive outcome measures at post-treatment \\
\hline $\begin{array}{l}\text { Emotion } \\
\text { perception }\end{array}$ & $\begin{array}{l}\text { Pictures of Facial Affect (POFA; Ekman \& Friesen, 1976) } \\
\text { Face Emotion Identification Test (FEIT; Kerr \& Neale, 1993) } \\
\text { Face Emotion Discrimination Test (FEDT; Kerr \& Neale, 1993) } \\
\text { Cambridge Mindreading Face-Voice Battery (Golan, Baron-Cohen \& Hill, 2006) } \\
\text { Reading the mind in the Voice Task Revised (Golan, Baron-Cohen, Hill \& Rutherford, } \\
2006 \text { ) } \\
\text { Reading the Mind in Film Task (Golan, Baron-Cohen, Hill \& Golan, 2006) } \\
\text { Emotion Recognition-40 (Lahera et al., 2013) } \\
\text { The Awareness of Social Inference Test-Part 1 (TASIT; McDonald et al., 2003; 2006) } \\
\text { Emotion Attribution Task (Blair \& Cipolotti, 2000; Mazza et al., 2007) } \\
\text { Bell-Lysaker Emotion Recognition Task (BLERT; Bell, Bryson, \& Lysaker, 1997) } \\
\text { Emotion Recognition Task (Höschel \& Irle, 2001) } \\
\text { Japanese and Caucasian Facial Expressions of Emotion (Matsumoto \& Ekman, } \\
\text { 1988) } \\
\text { Vienna Emotion Recognition Task (VERT-K; Pawelak, 2004) } \\
\text { Geneva Vocal Emotion Expression Stimulus (GVEESS; Banse \& Scherer, 1996) } \\
\text { Emotion Recognition Test-40 faces edition (Kohler et al., 2005) } \\
\text { Computerised test of facial emotion recognition (CFER; based on Tottenham, 1998) } \\
\text { Levels of Emotional Awareness Scale (LEAS; Lane, Quinlan, Schwartz, Walker \& } \\
\text { Zeitlin, 1990) } \\
\text { Facial Expression Same/Different Task (Green, Turner \& Thompson, 2004) } \\
\text { Facial Expression Naming Task (Hornak, Rolls \& Wade, 1996) } \\
\text { Facial Expression Matching Task (Croker \& McDonald, 2005) } \\
\text { Prosodic Emotion Labelling Task (Dimoska, McDonald, Pell, Tate \& James, 2010) } \\
\text { Diagnostic Analysis of Non-verbal Accuracy scale (DANVA 2; Nowicki \& Duke, } \\
\text { 1994) } \\
\text { Frankfurt Test and Training of Facial Affect Recognition (FEFA; Bölte et al., 2002) } \\
\text { NimStim Face Stimulus Set (Tottenham et al., 2009) } \\
\text { Emotion Recognition Questionnaire (Emorec, Bähler, 2012) } \\
\text { Emotional Inference from Stories Test (EIST, Neumann et al., 2012; Zupan, 2009) }\end{array}$ \\
\hline Social perception & $\begin{array}{l}\text { Social Perception Scale (SPS; Garcia et al., 2003) } \\
\text { Half-Profile of Non-verbal Sensitivity (PONS; Ambady et al., 1995; Rosenthal et al., } \\
\text { 1979). } \\
\text { Schema Component Sequencing Task-Revised (Vauth, Rüsch, Wirtz \& Corrigan, } \\
\text { 2004) } \\
\text { Social Situation Task (Blair \& Cipolotti, 2000) }\end{array}$ \\
\hline Theory of Mind & $\begin{array}{l}\text { Reading the Mind in the Eyes Task Revised (Baron-Cohen et al., 2001) } \\
\text { Theory of Mind Picture Sequencing Task and Questionnaire (PST; Brüne, 2003a, } \\
\text { 2003b, 2005) } \\
\text { Hinting Task (Corcoran et al., 1995) } \\
\text { TASIT-Part } 2 \text { \& } 3 \text { (McDonald et al., 2003; 2006) } \\
\text { Advanced Theory of Mind Scale (Blair \& Cipolotti, 2000; Happe, 1994) } \\
\text { False belief stories (Washing Machine Story and Wallpaper Story; Mazza et al., } \\
\text { 2007; Rowe, Bullock, Poley \& Morris, 2001) } \\
\text { Empathy Questionnaire (EQ; Baron-Cohen \& Wheelwright, 2004) } \\
\text { Unexpected Outcomes Test (UOT; Dyck, Ferguson \& Shochet, 2001; Bora, Eryavuz, } \\
\text { Kayahan, Sungu \& Veznedaroglu, 2008) } \\
\text { Nonverbal ToM-task (Kayser et al., 2007) } \\
\text { False belief/deception stories ('The Cigarettes, Happe, 1994; 'The Piggy Bank', } \\
\text { 'The Train Station', and 'The Coke', Frith \& Corcoran, 1996) } \\
\text { Interpersonal Reactivity Index (IRI; Davis, 1983) }\end{array}$ \\
\hline
\end{tabular}




\section{TABLE 1}

Continued

Social cognitive

domain Social cognitive outcome measures at post-treatment

Social functioning Social Functioning Scale (SFS; subscales social engagement \& interaction, Birchwood et al., 1990)

Mayer-Salovey-Caruso Emotional Intelligence Test (MSCEIT; subtest managing emotions, Mayer et al., 2002; 2003)

Social Cognition Profile (Hogarty \& Greenwald, 2006)

Behavioural Referenced Rating System of Intermediate Social Skills - Revised

(BRISS-R; Partner Directed Behaviour Scale and Personal Conversational Style

Scale, Wallander, Conger, \& Conger, 1985; Farrell, Rabinowitz, Wallander \&

Curran, 1985)

Life Skills Profile (LSP; subscale social contact, Rosen, Hadzi-Pavlovic \& Parker,

1989)

Personal and Social Performance Scale (PSP; subscale personal and social

relationships, Morosini et al., 2000; Hsieh et al, 2011)

Social Performance Survey Schedule (SPSS; Lowe \& Cautela, 1978)

Social Skills Performance Assessment (SSPA; Patterson et al., 2001)

Assertion Inventory (Al; subscale behaviour, Gambrill \& Richey, 1975; Martin et al., 2012)

Simulated Social Interaction Test (SSIT; Curran, 1982)

Social Role-play Task (Penn, Mueser, Doonan \& Nishith, 1995)

Observable Social Cognition, A Rating Scale (OSCARS; Healey, Roberts, Combs \& Penn, 2012)

Global Social Functioning Scale (GSFS; Cornblatt et al., 2007)

Social Communication Skills Questionnaire (SCSQ; McGann, Werven \& Douglas, 1997)

Brock Adaptive Functioning Questionnaire (BAFQ; socio-emotional scale, Dywan, Roden \& Murphy, 1995)

\section{Results}

\section{Results of the Search}

The initial systematic searches of the databases in 2013 yielded 3,676 hits. After a first screening based on titles and the removal of duplicates 341 records remained. Reasons for exclusion were, for example, the use of animal subjects or the evaluation of a pharmacological intervention. After independent screening of the 341 abstracts by authors RLR and EW, 75 full-text articles were selected for closer reading. Subsequently, additional searches for the years 2014 and 2015 were performed, resulting in 45 full-text articles that were assessed for eligibility. Last, the reference lists of the selected studies and relevant review articles were searched. This resulted in another 46 full-text articles that were assessed for eligibility. Of the 166 articles that were potentially eligible for inclusion, 41 studies were finally included. The majority of the studies was excluded because one or more of the inclusion criteria was not met $(k=122)$ and four studies were excluded due to overlap with other included studies.
In 13 studies, additional information for the meta-analysis was required and the corresponding authors were contacted by e-mail (Bechi et al., 2013, 2015; Combs et al., 2007; Eack et al., 2009; Fuentes, Garcia, Ruiz, Soler \& Roder, 2007; GilSanz et al., 2009; Hogarty et al., 2004; Horan et al., 2011; Lindenmayer et al., 2013; Mazza et al., 2010; Rocha \& Queirós, 2013; Roncone et al., 2004; Veltro et al., 2011). The requested information was received from the majority of the authors, with exception of three studies. One study had to be excluded due to missing information.

\section{Description of Included Studies}

Table 2 shows a detailed description of the study characteristics. Forty-one studies were included in the review and meta-analyses. The dates of publication ranged from 2003 to 2015. In the article of Golan and Baron-Cohen (2006), two studies were reported that had been performed in different patient samples. Therefore, both experiments were included as separate studies in the meta-analysis. The majority of studies $(k=33)$ used a randomised 
TABLE 2

Characteristics of the 41 Included Studies

\begin{tabular}{|c|c|c|c|c|c|c|c|c|c|c|c|c|c|c|c|}
\hline Study & Population & $\begin{array}{l}\text { Name of } \\
\text { intervention }\end{array}$ & $\begin{array}{l}\text { Type of } \\
\text { intervention }\end{array}$ & $\begin{array}{l}\text { PEDro-P } \\
\text { score } \\
(0-10)\end{array}$ & $\begin{array}{l}\text { Intervention } \\
\text { target(s) }\end{array}$ & $\begin{array}{l}\text { Control } \\
\text { group }\end{array}$ & $\begin{array}{l}\text { Sample size } \\
(\mathrm{Nt} / \mathrm{Nc})\end{array}$ & $\%$ Male & Design & Age of patients & $\begin{array}{l}\text { Duration/ } \\
\text { dose of } \\
\text { intervention }\end{array}$ & $\begin{array}{l}\text { Group } \\
\text { size }\end{array}$ & $\begin{array}{l}\text { Outcome } \\
\text { measures }\end{array}$ & $\begin{array}{l}\text { Follow- } \\
\text { up }\end{array}$ & Main findings \\
\hline $\begin{array}{l}\text { Bechi et al. } \\
2012\end{array}$ & SSD & $\begin{array}{l}\text { Social } \\
\text { Cognitive } \\
\text { Training } \\
\text { (SCT) } \\
\text { combined } \\
\text { with } \\
\text { cognitive } \\
\text { rehabilita- } \\
\text { tion training } \\
\text { (CRT) }\end{array}$ & B & 4 & $\begin{array}{l}E P, \text { ToM, and } \\
\text { neurocogni- } \\
\text { tion }\end{array}$ & $\begin{array}{l}\text { Time- } \\
\text { matched } \\
\text { control } \\
\text { group (NI) }\end{array}$ & $\begin{array}{l}49 \\
(27 / 22)\end{array}$ & $68 / 67$ & $\begin{array}{l}\text { Quasi- } \\
\text { experiment }\end{array}$ & $\begin{array}{l}\text { SCT: } 37.14 \\
\text { (10.02) } \\
\text { NI: } 40.20 \text { (8.99) }\end{array}$ & $\begin{array}{l}12 \text { sess./12 } \\
\text { h./12 wks. } \\
\text { SCT } \\
\text { combined } \\
\text { with } \\
24 \\
\text { sess./24h./12 } \\
\text { wks. CRT }\end{array}$ & 5 & $\begin{array}{l}\text { - POFA (EP) } \\
\text { - PST } \\
\text { Sequencing } \\
\text { (ToM) } \\
\text { - PST Ques- } \\
\text { tionnaire } \\
\text { (ToM) }\end{array}$ & - & $\begin{array}{l}\text { Improvement } \\
\text { on ToM } \\
\text { Question- } \\
\text { naire. }\end{array}$ \\
\hline $\begin{array}{l}\text { Bechi et al. } \\
2013\end{array}$ & SSD & $\begin{array}{l}\text { ToM- } \\
\text { Intervention } \\
\text { (ToMI) } \\
\text { combined } \\
\text { with CRT }\end{array}$ & B & 5 & $\begin{array}{l}\text { ToM, SP, and } \\
\text { neurocogni- } \\
\text { tion }\end{array}$ & $\begin{array}{l}\text { Active } \\
\text { control } \\
\text { group with } \\
\text { cognitive } \\
\text { remediation } \\
\text { therapy } \\
(\mathrm{NSCl})\end{array}$ & $\begin{array}{l}30 \\
(19 / 11)\end{array}$ & $42 / 54$ & RCT & $\begin{array}{l}\text { ToMI: } 37.68 \\
(8.42) \\
\text { NSCl: } 37.73 \\
(15.45)\end{array}$ & $\begin{array}{l}18 \text { sess./18 } \\
\text { h/9 wks. } \\
\text { SCT } \\
\text { combined } \\
\text { with } \\
36 \\
\text { sess./36h./12 } \\
\text { wks. CRT }\end{array}$ & 5 & $\begin{array}{l}\text { - PST Total } \\
\text { (ToM) }\end{array}$ & - & $\begin{array}{l}\text { Improvement } \\
\text { on PST. }\end{array}$ \\
\hline $\begin{array}{l}\text { Bechi et al. } \\
2015\end{array}$ & SSD & $\begin{array}{l}\text { ToMl } \\
\text { combined } \\
\text { with CRT }\end{array}$ & B & 6 & $\begin{array}{l}\text { ToM, SP, and } \\
\text { neurocogni- } \\
\text { tion }\end{array}$ & $\begin{array}{l}\text { Active } \\
\text { control } \\
\text { group with } \\
\text { cognitive } \\
\text { remediation } \\
\text { therapy } \\
(\mathrm{NSCl})\end{array}$ & $\begin{array}{l}51 \\
(32 / 19)\end{array}$ & $\begin{array}{l}53.13 / \\
52.63\end{array}$ & RCT & $\begin{array}{l}\text { ToMI: } 40.34 \\
\text { (10.33) } \\
\text { NSCl: } 37.21 \\
(12.45)\end{array}$ & $\begin{array}{l}18 \\
\text { sess./18h./9 } \\
\text { wks. ToMl } \\
\text { combined } \\
\text { with } 24 \\
\text { sess./24 } \\
\text { h/12 wks. } \\
\text { CRT }\end{array}$ & 5 & $\begin{array}{l}\text { - PST Total } \\
\text { (ToM) }\end{array}$ & - & $\begin{array}{l}\text { Improvement } \\
\text { on PST. }\end{array}$ \\
\hline $\begin{array}{l}\text { Bölte et al. } \\
2006\end{array}$ & ASD & $\begin{array}{l}\text { Frankfurt } \\
\text { Test and } \\
\text { Training of } \\
\text { Facial Affect } \\
\text { Recognition } \\
\text { (FEFA) }\end{array}$ & $\mathrm{T}$ & 4 & EP & $\begin{array}{l}\text { Ongoing } \\
\text { inpatient } \\
\text { treatment } \\
(\mathrm{TAU})\end{array}$ & $\begin{array}{l}10 \\
(5 / 5)\end{array}$ & 91 & RCT & $\begin{array}{l}\text { FEFA: } 29.4 \\
(5.9) \\
\text { TAU: } 25.8(8.0)\end{array}$ & $\begin{array}{l}5 \text { sess. } / 10 \\
\text { h/5 wks. }\end{array}$ & $\begin{array}{l}\text { Unkn- } \\
\text { own }\end{array}$ & $\begin{array}{l}\text { - FEFA Face } \\
\text { (EP) } \\
\text { - FEFA Eyes } \\
\text { (EP) }\end{array}$ & - & $\begin{array}{l}\text { Improvement } \\
\text { on FEFA Face } \\
\text { and Eyes. }\end{array}$ \\
\hline
\end{tabular}


TABLE 2

Continued

\begin{tabular}{|c|c|c|c|c|c|c|c|c|c|c|c|c|c|c|c|}
\hline Study & Population & $\begin{array}{l}\text { Name of } \\
\text { intervention }\end{array}$ & $\begin{array}{l}\text { Type of } \\
\text { intervention }\end{array}$ & $\begin{array}{l}\text { PEDro-P } \\
\text { score } \\
(0-10)\end{array}$ & $\begin{array}{l}\text { Intervention- } \\
\text { target(s) }\end{array}$ & $\begin{array}{l}\text { Control } \\
\text { group }\end{array}$ & $\begin{array}{l}\text { Sample size } \\
(\mathrm{Nt} / \mathrm{Nc})\end{array}$ & $\%$ Male & Design & Age of patients & $\begin{array}{l}\text { Duration/ } \\
\text { dose of } \\
\text { intervention }\end{array}$ & $\begin{array}{l}\text { Group } \\
\text { size }\end{array}$ & $\begin{array}{l}\text { Outcome } \\
\text { measures }\end{array}$ & $\begin{array}{l}\text { Follow- } \\
\text { up }\end{array}$ & Main findings \\
\hline $\begin{array}{l}\text { Bornhofen \& } \\
\text { McDonald } \\
\text { 2008a }\end{array}$ & $A B I$ & $\begin{array}{l}\text { Emotion } \\
\text { Perception } \\
\text { Programme } \\
\text { (EPP) }\end{array}$ & T & 6 & EP & $\begin{array}{l}\text { Waitlist } \\
\text { control } \\
\text { group (NI) }\end{array}$ & $11(5 / 6)$ & - & RCT & $\begin{array}{l}\text { EPP: } 29.2 \\
\text { (4.49) } \\
\text { Nl: } 43.5(14.43)\end{array}$ & $\begin{array}{l}16 \text { sess./24 } \\
\text { h./8 wks. }\end{array}$ & $2-3$ & $\begin{array}{l}\text { - FENT (EP) } \\
\text { - FEMT (EP) } \\
\text {-TASIT-1 } \\
\text { (EP) } \\
\text {-TASIT-2 \& } 3 \\
\text { (ToM) }\end{array}$ & $1 \mathrm{mo}$. & $\begin{array}{l}\text { Improvement } \\
\text { on TASIT-1 } \\
\text { and TASIT-3. }\end{array}$ \\
\hline $\begin{array}{l}\text { Bornhofen \& } \\
\text { McDonald } \\
\text { 2008b }\end{array}$ & $A B I$ & $\begin{array}{l}\text { Emotion } \\
\text { Perception } \\
\text { Treatment- } \\
\text { Self- } \\
\text { instruction } \\
\text { Training } \\
\text { (SIT) }\end{array}$ & T & 6 & EP & $\begin{array}{l}\text { Waitlist } \\
\text { control } \\
\text { group (NI) }\end{array}$ & $\begin{array}{l}10 \\
(5 / 5)\end{array}$ & - & RCT & $\begin{array}{l}\text { SIT: } 35.40 \\
(14.01) \\
\text { NI: } 31.20 \\
(16.83)\end{array}$ & $\begin{array}{l}10 \text { sess. } / 25 \\
\text { h./10 wks. }\end{array}$ & $2-3$ & $\begin{array}{l}\text { - FES/DT } \\
\text { (EP) } \\
\text { - FENT (EP) } \\
\text { - FEMT (EP) } \\
\text { - TASIT-1 } \\
\text { (EP) } \\
\text { - TASIT-2 \& } \\
\text { 3 ToM) }\end{array}$ & 6 mos. & $\begin{array}{l}\text { Improvement } \\
\text { on FEMT and } \\
\text { TASIT-2. }\end{array}$ \\
\hline $\begin{array}{l}\text { Combs et al. } \\
2007\end{array}$ & SSD & $\begin{array}{l}\text { Social } \\
\text { Cognition } \\
\text { and } \\
\text { Interaction } \\
\text { Training } \\
\text { (SCIT) }\end{array}$ & C & 5 & EP and ToM & $\begin{array}{l}\text { Coping skills } \\
\text { group } \\
\text { (NSCl) }\end{array}$ & $28(18 / 10)$ & $67 / 90$ & $\begin{array}{l}\text { Quasi- } \\
\text { experiment }\end{array}$ & $\begin{array}{l}\text { SCIT: } 41.3 \\
(11.2) \\
\text { NSCI: } 44.0 \\
(10.6)\end{array}$ & $\begin{array}{l}18 \text { sess. } / 18 \\
\text { h./18 wks. }\end{array}$ & 8-10 & $\begin{array}{l}\text { - FEIT (EP) } \\
\text { - FEDT (EP) } \\
\text { - SPS, } \\
\text { stimulus } \\
\text { identification } \\
\text { (SP) } \\
\text { - HT (ToM) } \\
\text { - SFS, (SF) }\end{array}$ & - & $\begin{array}{l}\text { Improvement } \\
\text { on all } \\
\text { outcome } \\
\text { measures. }\end{array}$ \\
\hline
\end{tabular}


TABLE 2

Continued

\begin{tabular}{|c|c|c|c|c|c|c|c|c|c|c|c|c|c|c|c|}
\hline Study & Population & $\begin{array}{l}\text { Name of } \\
\text { intervention }\end{array}$ & $\begin{array}{l}\text { Type of } \\
\text { intervention }\end{array}$ & $\begin{array}{l}\text { PEDro-P } \\
\text { score } \\
(0-10)\end{array}$ & $\begin{array}{l}\text { Intervention- } \\
\text { target(s) }\end{array}$ & $\begin{array}{l}\text { Control } \\
\text { group }\end{array}$ & $\begin{array}{l}\text { Sample size } \\
(\mathrm{Nt} / \mathrm{Nc})\end{array}$ & $\%$ Male & Design & Age of patients & $\begin{array}{l}\text { Duration/ } \\
\text { dose of } \\
\text { intervention }\end{array}$ & $\begin{array}{l}\text { Group } \\
\text { size }\end{array}$ & $\begin{array}{l}\text { Outcome } \\
\text { measures }\end{array}$ & $\begin{array}{l}\text { Follow- } \\
\text { up }\end{array}$ & Main findings \\
\hline $\begin{array}{l}\text { Combs et al. } \\
2008\end{array}$ & SSD & $\begin{array}{l}\text { Attentional } \\
\text { Shaping } \\
\text { (AS) }\end{array}$ & T & 5 & EP & $\begin{array}{l}\text { Repeated } \\
\text { practise (NI) }\end{array}$ & $40(20 / 20)$ & 65 & RCT & $\begin{array}{l}\text { AS/NI: } 38.7 \\
(13.7)\end{array}$ & 1 sess. & 1 & - FEIT (EP) & 1 week & $\begin{array}{l}\text { Improvement } \\
\text { on FEIT. }\end{array}$ \\
\hline $\begin{array}{l}\text { Eack et al. } \\
2009\end{array}$ & SSD & $\begin{array}{l}\text { Cognitive } \\
\text { Enhance- } \\
\text { ment } \\
\text { Therapy } \\
\text { (CET) }\end{array}$ & B & 6 & $\begin{array}{l}\text { ToM, SP, SF, } \\
\text { and neu- } \\
\text { rocognition }\end{array}$ & $\begin{array}{l}\text { Enriched } \\
\text { Supportive } \\
\text { Therapy } \\
\text { (NSCl) }\end{array}$ & $58(31 / 27)$ & 69 & RCT & $\begin{array}{l}\text { CET/NSCl: } \\
25.92(6.31)\end{array}$ & $\begin{array}{l}60 \text { sess./60 } \\
\text { h./104 wks. } \\
\text { neurocogni- } \\
\text { tive training } \\
\text { combined } \\
\text { with } 45 \\
\text { sess./67.5h./ } \\
104 \text { wks. } \\
\text { social } \\
\text { cognitive } \\
\text { training }\end{array}$ & $\begin{array}{l}\text { Unkn- } \\
\text { own }\end{array}$ & $\begin{array}{l}\text { - SC Profile } \\
\text { (SF) } \\
\text { - MSCEIT } \\
\text { (SF) }\end{array}$ & - & $\begin{array}{l}\text { Improvement } \\
\text { on both } \\
\text { outcome } \\
\text { measures. }\end{array}$ \\
\hline $\begin{array}{l}\text { Fernandez- } \\
\text { Gonzalo } \\
\text { et al. } 2015\end{array}$ & SSD & $\begin{array}{l}\text { Neuro } \\
\text { Personal } \\
\text { Training- } \\
\text { Mental } \\
\text { Health } \\
\text { (NPT-MH) }\end{array}$ & B & 5 & $\begin{array}{l}E P, T o M \text {, and } \\
\text { neurocogni- } \\
\text { tion }\end{array}$ & $\begin{array}{l}\text { Non- } \\
\text { specific } \\
\text { computer } \\
\text { training (NI) }\end{array}$ & $40(21 / 19)$ & $60.7 / 68$ & RCT & $\begin{array}{l}\text { NPT-MH: } 30.9 \\
\text { (5.9) } \\
\text { Control: } 30.02 \\
\text { (7.4) }\end{array}$ & $\begin{array}{l}+/-36 \\
\text { sess./36 } \\
\text { h./18 wks. }\end{array}$ & 6 & $\begin{array}{l}\text { - POFA (EP) } \\
\text { - False- } \\
\text { belief/deception } \\
\text { stories (ToM) } \\
\text { - HT (ToM) } \\
\text { - RMET (EP) }\end{array}$ & - & $\begin{array}{l}\text { Improvement } \\
\text { on POFA. }\end{array}$ \\
\hline $\begin{array}{l}\text { Garcia et al. } \\
2003\end{array}$ & SSD & $\begin{array}{l}\text { Social } \\
\text { Perception } \\
\text { subpro- } \\
\text { gramme of } \\
\text { Integrated } \\
\text { Psychologi- } \\
\text { cal Therapy } \\
\text { (IPT) }\end{array}$ & T & 4 & SP & $\begin{array}{l}\text { Not } \\
\text { specified }\end{array}$ & $20(11 / 9)$ & $82 / 56$ & RCT & $\begin{array}{l}\text { IPT: } 40.45 \\
\text { (7.10) } \\
\text { Control: } 36.88 \\
(8.10)\end{array}$ & $\begin{array}{l}21 \\
\text { sess./18.5h./ } \\
12 \text { wks. }\end{array}$ & $5-6$ & - SPS (SP) & - & $\begin{array}{l}\text { Improvement } \\
\text { on SPS. }\end{array}$ \\
\hline $\begin{array}{l}\text { Gil-Sanz } \\
\text { et al. } 2009\end{array}$ & SSD & $\begin{array}{l}\text { Social } \\
\text { Cognition } \\
\text { Training } \\
\text { Program } \\
\text { (SCTP) }\end{array}$ & c & 4 & $E P$ and $S P$ & TAU & $14(7 / 7)$ & $57 / 43$ & RCT & $\begin{array}{l}\text { SCTP: } 33.29 \\
(8.36) \\
\text { TAU: } 41.43 \\
(9.03)\end{array}$ & $\begin{array}{l}20 \\
\text { sess./15h./10 } \\
\text { wks. }\end{array}$ & $\begin{array}{l}\text { Unkn- } \\
\text { own }\end{array}$ & $\begin{array}{l}\text { - CFER (EP) } \\
\text { - SPS (SP) }\end{array}$ & - & $\begin{array}{l}\text { Improvement } \\
\text { on SPS } \\
\text { interpretation } \\
\text { and } \\
\text { allocation of } \\
\text { title. }\end{array}$ \\
\hline
\end{tabular}




\section{TABLE 2}

Continued

\begin{tabular}{|c|c|c|c|c|c|c|c|c|c|c|c|c|c|c|c|}
\hline Study & Population & $\begin{array}{l}\text { Name of } \\
\text { intervention }\end{array}$ & $\begin{array}{l}\text { Type of } \\
\text { intervention }\end{array}$ & $\begin{array}{l}\text { PEDro-P } \\
\text { score } \\
(0-10)\end{array}$ & $\begin{array}{l}\text { Intervention- } \\
\text { target(s) }\end{array}$ & $\begin{array}{l}\text { Control } \\
\text { group }\end{array}$ & $\begin{array}{l}\text { Sample size } \\
(\mathrm{Nt} / \mathrm{Nc})\end{array}$ & $\%$ Male & Design & Age of patients & $\begin{array}{l}\text { Duration/ } \\
\text { dose of } \\
\text { intervention }\end{array}$ & $\begin{array}{l}\text { Group } \\
\text { size }\end{array}$ & $\begin{array}{l}\text { Outcome } \\
\text { measures }\end{array}$ & $\begin{array}{l}\text { Follow- } \\
\text { up }\end{array}$ & Main findings \\
\hline $\begin{array}{l}\text { Gil-Sanz } \\
\text { et al. } 2014\end{array}$ & SSD & SCTP & C & 5 & $\begin{array}{l}\mathrm{EP}, \mathrm{ToM} \text {, and } \\
\mathrm{SP}\end{array}$ & $\begin{array}{l}\text { Attention } \\
\text { and memory } \\
\text { exercises } \\
\text { (NSCl) }\end{array}$ & $44(20 / 24)$ & $40 / 66.67$ & RCT & $\begin{array}{l}\text { SCTP: } 37.05 \\
\text { (6.43) } \\
\text { Control: } 43.83 \\
(9.77)\end{array}$ & $\begin{array}{l}28 \text { sess./ } 21 \\
\text { h./28 wks. }\end{array}$ & 10 & $\begin{array}{l}\text { - HT (ToM) } \\
\text { - NimStimFace } \\
\text { Stimulus Set } \\
\text { Total Score } \\
\text { (EP) }\end{array}$ & - & $\begin{array}{l}\text { Improvement } \\
\text { on HT and } \\
\text { NimStimFace } \\
\text { Stiumulus } \\
\text { Set. }\end{array}$ \\
\hline $\begin{array}{l}\text { Golan \& } \\
\text { Baron- } \\
\text { Cohen 2006, } \\
\text { exp. } 1\end{array}$ & ASD & $\begin{array}{l}\text { Mind } \\
\text { Reading } \\
\text { (MR) }\end{array}$ & $\mathrm{T}$ & 6 & EP & $\begin{array}{l}\text { No } \\
\text { intervention } \\
\text { (NI) }\end{array}$ & $41(19 / 22)$ & 74/77 & RCT & $\begin{array}{l}\text { MR: } 30.5 \text { (10.3) } \\
\mathrm{Nl}: 30.9(11.2)\end{array}$ & $\begin{array}{l}20 \mathrm{~h} . / 10 \\
\text { wks. }\end{array}$ & 1 & $\begin{array}{l}\text { - CAM Face } \\
\text { (EP) } \\
\text { - CAM Voice } \\
\text { (EP) } \\
\text { - CAM } \\
\text { Emotion (EP) } \\
\text { - RMET (EP) } \\
\text { - RMVT (EP) } \\
\text { - RMFT (EP) }\end{array}$ & - & $\begin{array}{l}\text { Improvement } \\
\text { on all CAM } \\
\text { measures. }\end{array}$ \\
\hline $\begin{array}{l}\text { Golan \& } \\
\text { Baron- } \\
\text { Cohen 2006, } \\
\text { exp. } 2\end{array}$ & ASD & MR & $\mathrm{T}$ & 4 & EP & $\begin{array}{l}\text { Social skills } \\
\text { training } \\
\text { (SCI) }\end{array}$ & $26(13 / 13)$ & $92 / 77$ & $\begin{array}{l}\text { Quasi- } \\
\text { experiment }\end{array}$ & $\begin{array}{l}\text { MR: } 25.5 \text { (9.3) } \\
\text { SCI: } 24.4(6.4)\end{array}$ & $\begin{array}{l}20 \mathrm{~h} . / 10 \\
\text { wks. } \\
\text { individual } \\
\text { training } \\
\text { combined } \\
\text { with } 10 \\
\text { sess. } / 10 \\
\text { wks. } \\
\text { group } \\
\text { training }\end{array}$ & $1 / 6$ & $\begin{array}{l}\text { - CAM Face } \\
\text { (EP) } \\
\text { - CAM Voice } \\
\text { (EP) } \\
\text { - CAM } \\
\text { Emotion (EP) } \\
\text { - RMET (EP) } \\
\text { - RMVT (EP) } \\
\text { - RMFT (EP) }\end{array}$ & - & $\begin{array}{l}\text { Improvement } \\
\text { on CAM } \\
\text { Voice, CAM } \\
\text { Emotion, and } \\
\text { RMET. }\end{array}$ \\
\hline
\end{tabular}


TABLE 2

Continued

\begin{tabular}{|c|c|c|c|c|c|c|c|c|c|c|c|c|c|c|c|}
\hline Study & Population & $\begin{array}{l}\text { Name of } \\
\text { intervention }\end{array}$ & $\begin{array}{l}\text { Type of } \\
\text { intervention }\end{array}$ & $\begin{array}{l}\text { PEDro-P } \\
\text { score } \\
(0-10)\end{array}$ & $\begin{array}{l}\text { Intervention- } \\
\text { target(s) }\end{array}$ & $\begin{array}{l}\text { Control } \\
\text { group }\end{array}$ & $\begin{array}{l}\text { Sample size } \\
(\mathrm{Nt} / \mathrm{Nc})\end{array}$ & $\%$ Male & Design & Age of patients & $\begin{array}{l}\text { Duration/ } \\
\text { dose of } \\
\text { intervention }\end{array}$ & $\begin{array}{l}\text { Group } \\
\text { size }\end{array}$ & $\begin{array}{l}\text { Outcome } \\
\text { measures }\end{array}$ & $\begin{array}{l}\text { Follow- } \\
\text { up }\end{array}$ & Main findings \\
\hline $\begin{array}{l}\text { Hogarty } \\
\text { et al. } 2004\end{array}$ & SSD & CET & B & 5 & $\begin{array}{l}\text { ToM, SP, SF, } \\
\text { and neu- } \\
\text { rocognition }\end{array}$ & $\begin{array}{l}\text { Enriched } \\
\text { Supportive } \\
\text { Therapy } \\
\text { (NSCl) }\end{array}$ & $113(63 / 50)$ & 59 & RCT & $37.3(8.9)$ & $\begin{array}{l}75 \mathrm{~h} . / 104 \\
\text { wks. neu- } \\
\text { rocognitive } \\
\text { training } \\
\text { combined } \\
\text { with } \\
56 \text { sess. } / 84 \\
\text { h./104 wks. } \\
\text { social } \\
\text { cognitive } \\
\text { training }\end{array}$ & 6 & $\begin{array}{l}\text { - SC Profile } \\
\text { (SF) }\end{array}$ & $\begin{array}{l}\text { 3-year } \\
\text { (pub- } \\
\text { lished } \\
\text { in } \\
\text { Hogarty } \\
\text { et al. } \\
\text { 2006) }\end{array}$ & $\begin{array}{l}\text { Improvement } \\
\text { on SC Profile } \\
\text { subscales } \\
\text { Tolerant, } \\
\text { Perceptive, } \\
\text { and Self- } \\
\text { confident. }\end{array}$ \\
\hline $\begin{array}{l}\text { Horan et al. } \\
2009\end{array}$ & SSD & $\begin{array}{l}\text { Social } \\
\text { Cognitive } \\
\text { Skills } \\
\text { Training } \\
\text { (SCST) }\end{array}$ & C & 5 & $\begin{array}{l}\text { EP, ToM, and } \\
\text { SP }\end{array}$ & $\begin{array}{l}\text { Illness self- } \\
\text { management } \\
\text { and relapse } \\
\text { prevention } \\
\text { skills } \\
\text { training } \\
\text { (TAU) }\end{array}$ & 31 (15/16) & $87 / 100$ & RCT & $\begin{array}{l}\text { SCST: } 50.7 \\
(5.8) \\
\text { NSCl: } 45.9 \\
(7.5)\end{array}$ & $\begin{array}{l}12 \text { sess./12 } \\
\text { h./6 wks. }\end{array}$ & 6 & $\begin{array}{l}\text { - FEIT (EP) } \\
\text { - PONS (SP) } \\
\text {-TASIT-3 } \\
\text { (ToM) }\end{array}$ & - & $\begin{array}{l}\text { Improvement } \\
\text { on FEIT. }\end{array}$ \\
\hline $\begin{array}{l}\text { Horan et al. } \\
2011\end{array}$ & SSD & SCST & C & 5 & $\begin{array}{l}\text { EP, ToM, and } \\
\text { SP }\end{array}$ & $\begin{array}{l}\text { Standard } \\
\text { Illness Man- } \\
\text { agement } \\
\text { Training } \\
(\mathrm{TAU})\end{array}$ & $33(15 / 18)$ & $94 / 79$ & RCT & $\begin{array}{l}\text { SCST: } 51.0 \\
\text { (7.1) } \\
\text { TAU: } 45.1 \\
(11.2)\end{array}$ & $\begin{array}{l}24 \text { sess./24 } \\
\text { h./12 wks. }\end{array}$ & $6-8$ & $\begin{array}{l}\text { - FEIT (EP) } \\
\text { - MSCEIT } \\
\text { (SF) } \\
\text { - PONS (SP) } \\
\text { - TASIT-3 } \\
\text { (ToM) }\end{array}$ & - & $\begin{array}{l}\text { Improvement } \\
\text { on FEIT. }\end{array}$ \\
\hline $\begin{array}{l}\text { Kayser et al. } \\
2007\end{array}$ & SSD & $\begin{array}{l}\text { ToM Video } \\
\text { Training } \\
\text { (ToM-VT) }\end{array}$ & $\mathrm{T}$ & 5 & ToM & TAU & $14(8 / 6)$ & $75 / 83$ & RCT & $\begin{array}{l}\text { ToM-VT: } 32.4 \\
\text { (9.4) } \\
\text { TAU: } 38.2(9.3)\end{array}$ & $\begin{array}{l}2 \\
\text { sess./2h./2 } \\
\text { wks. }\end{array}$ & $6-8$ & $\begin{array}{l}\text { - Non-verbal } \\
\text { ToM task } \\
\text { (ToM) }\end{array}$ & - & $\begin{array}{l}\text { Improvement } \\
\text { on Nonverbal } \\
\text { ToM. }\end{array}$ \\
\hline
\end{tabular}


TABLE 2

Continued

\begin{tabular}{|c|c|c|c|c|c|c|c|c|c|c|c|c|c|c|c|}
\hline Study & Population & $\begin{array}{l}\text { Name of } \\
\text { intervention }\end{array}$ & $\begin{array}{l}\text { Type of } \\
\text { intervention }\end{array}$ & $\begin{array}{l}\text { PEDro-P } \\
\text { score } \\
(0-10)\end{array}$ & $\begin{array}{l}\text { Intervention- } \\
\text { target(s) }\end{array}$ & $\begin{array}{l}\text { Control } \\
\text { group }\end{array}$ & $\begin{array}{l}\text { Sample size } \\
(\mathrm{Nt} / \mathrm{Nc})\end{array}$ & $\%$ Male & Design & Age of patients & $\begin{array}{l}\text { Duration/ } \\
\text { dose of } \\
\text { intervention }\end{array}$ & $\begin{array}{l}\text { Group } \\
\text { size }\end{array}$ & $\begin{array}{l}\text { Outcome } \\
\text { measures }\end{array}$ & $\begin{array}{l}\text { Follow- } \\
\text { up }\end{array}$ & Main findings \\
\hline $\begin{array}{l}\text { Lahera et al. } \\
2013\end{array}$ & $\begin{array}{l}\text { Bipolar \& } \\
\text { schizoaf- } \\
\text { fective } \\
\text { disorder }\end{array}$ & SCIT & C & 3 & EP and ToM & TAU & $37(21 / 16)$ & $24 / 50$ & $\begin{array}{l}\text { Quasi- } \\
\text { experiment }\end{array}$ & $\begin{array}{l}\text { SCIT: } 40.6(8.2) \\
\text { TAU: } 37.4 \\
\text { (12.7) }\end{array}$ & $\begin{array}{l}18 \text { sess./18 } \\
\text { h./18-24 } \\
\text { wks. }\end{array}$ & $\begin{array}{l}\text { Max. } \\
12\end{array}$ & $\begin{array}{l}\text { - FEIT (EP) } \\
\text { - FEDT (EP) } \\
\text { - ER-40 (EP) } \\
\text { - HT (ToM) }\end{array}$ & - & $\begin{array}{l}\text { Improvement } \\
\text { on all } \\
\text { measures. }\end{array}$ \\
\hline $\begin{array}{l}\text { Lindenmayer } \\
\text { et al. } 2013\end{array}$ & SSD & $\begin{array}{l}\text { Cognitive } \\
\text { remediation } \\
\text { (CR) } \\
\text { combined } \\
\text { with Mind } \\
\text { Reading: } \\
\text { Interactive } \\
\text { Guide to } \\
\text { Emotions } \\
\text { (MRIGE) }\end{array}$ & B & 6 & $\begin{array}{l}\text { EP and neu- } \\
\text { rocognition }\end{array}$ & $\begin{array}{l}\text { Cognitive } \\
\text { remediation } \\
\text { (NSCl) }\end{array}$ & $59(32 / 27)$ & 81 & RCT & $\begin{array}{l}\text { MRIGE: } 43.95 \\
\text { (11.12) } \\
\text { NSCl: } 42.48 \\
(9.09)\end{array}$ & $\begin{array}{l}\text { 24h./12wks. } \\
\text { CR } \\
\text { combined } \\
\text { with } \\
\text { 12h./12 } \\
\text { wks. MRIGE }\end{array}$ & $\begin{array}{l}\text { Unkn- } \\
\text { own }\end{array}$ & $\begin{array}{l}\text { - FEIT (EP) } \\
\text { - FEDT (EP) } \\
\text { - MSCEIT } \\
\text { (SF) }\end{array}$ & - & $\begin{array}{l}\text { Improvement } \\
\text { on all } \\
\text { measures. }\end{array}$ \\
\hline $\begin{array}{l}\text { Mazza et al. } \\
2010\end{array}$ & SSD & $\begin{array}{l}\text { Emotion } \\
\text { and ToM } \\
\text { Imitation } \\
\text { Training } \\
\text { (ETIT) }\end{array}$ & C & 6 & EP and ToM & $\begin{array}{l}\text { Problem } \\
\text { Solving Skill } \\
\text { Training } \\
\text { (NSCl) }\end{array}$ & $23(16 / 17)$ & 59 & RCT & $\begin{array}{l}\text { ETIT: } 24.37 \\
\text { (2.12) } \\
\text { NSCl: } 24.71 \\
(2.17)\end{array}$ & $\begin{array}{l}24 \text { sess./20 } \\
\text { h./12 wks. }\end{array}$ & $\begin{array}{l}\text { Unkn- } \\
\text { own }\end{array}$ & $\begin{array}{l}\text { - PSP (SF) } \\
\text { - Advanced } \\
\text { ToM-scale } \\
\text { (ToM) } \\
\text { - EAT (EP) } \\
\text { - EQ (ToM) }\end{array}$ & - & $\begin{array}{l}\text { Improvement } \\
\text { on all } \\
\text { measures. }\end{array}$ \\
\hline
\end{tabular}


TABLE 2

Continued

\begin{tabular}{|c|c|c|c|c|c|c|c|c|c|c|c|c|c|c|c|}
\hline Study & Population & $\begin{array}{l}\text { Name of } \\
\text { intervention }\end{array}$ & $\begin{array}{l}\text { Type of } \\
\text { intervention }\end{array}$ & $\begin{array}{l}\text { PEDro-P } \\
\text { score } \\
(0-10)\end{array}$ & $\begin{array}{l}\text { Intervention- } \\
\text { target(s) }\end{array}$ & $\begin{array}{l}\text { Control } \\
\text { group }\end{array}$ & $\begin{array}{l}\text { Sample size } \\
(\mathrm{Nt} / \mathrm{Nc})\end{array}$ & $\%$ Male & Design & Age of patients & $\begin{array}{l}\text { Duration/ } \\
\text { dose of } \\
\text { intervention }\end{array}$ & $\begin{array}{l}\text { Group } \\
\text { size }\end{array}$ & $\begin{array}{l}\text { Outcome } \\
\text { measures }\end{array}$ & $\begin{array}{l}\text { Follow- } \\
\text { up }\end{array}$ & Main findings \\
\hline $\begin{array}{l}\text { McDonald } \\
\text { et al. } 2008\end{array}$ & $\mathrm{ABI}$ & $\begin{array}{l}\text { Social Skills } \\
\text { Treatment } \\
\text { (SST) }\end{array}$ & B & 5 & $\begin{array}{l}\text { EP, ToM, SF } \\
\text { and social } \\
\text { skills }\end{array}$ & $\begin{array}{l}\text { Waitlist } \\
\text { control } \\
\text { group (NI) }\end{array}$ & $26(13 / 13)$ & 77 & $\begin{array}{l}\text { Quasi- } \\
\text { experiment }\end{array}$ & $\begin{array}{l}\text { SST: } 35.5 \\
\text { (11.3) } \\
\text { NI: } 35.3(11.6)\end{array}$ & $\begin{array}{l}12 \text { sess./36 } \\
\text { h./12 wks. } \\
\text { (group) } \\
\text { combined } \\
\text { with } \\
12 \text { sess./12 } \\
\text { h./12wks. } \\
\text { (individual) }\end{array}$ & $3-5$ & $\begin{array}{l}\text { - BRISS-R } \\
\text { PDBS (SF) } \\
\text { - BRISS-R } \\
\text { PCSS (SF) } \\
\text { - TASIT-1 } \\
\text { (EP) } \\
\text { - TASIT-2 \& } \\
3 \text { (ToM) } \\
\text { - SPSS (SF) }\end{array}$ & - & $\begin{array}{l}\text { Improvement } \\
\text { on BRISS-R } \\
\text { PDBS. }\end{array}$ \\
\hline $\begin{array}{l}\text { McDonald } \\
\text { et al. } 2013\end{array}$ & $\mathrm{ABI}$ & $\begin{array}{l}\text { Remediation } \\
\text { Programme } \\
\text { for } \\
\text { Perception } \\
\text { of Affective } \\
\text { Prosody } \\
\text { (RPPAP) }\end{array}$ & $\mathrm{T}$ & 7 & EP & $\begin{array}{l}\text { Waitlist } \\
\text { control } \\
\text { group (NI) }\end{array}$ & $20(10 / 10)$ & $60 / 90$ & RCT & $\begin{array}{l}\text { RPPAP/NI: } \\
45.62(11.26) \\
\text { RPPAP: } 44.54 \\
\text { NI: } 46.64\end{array}$ & $\begin{array}{l}3 \text { sess. } / 6 \\
\text { h./2 wks. }\end{array}$ & 2 & $\begin{array}{l}\text {-TASIT-1 } \\
\text { (EP) } \\
\text {-Prosodic } \\
\text { Emotion } \\
\text { Labelling } \\
\text { Task (EP) }\end{array}$ & $1 \mathrm{mo}$. & $\begin{array}{l}\text { No } \\
\text { improvement } \\
\text { on the } \\
\text { outcome } \\
\text { measures. }\end{array}$ \\
\hline $\begin{array}{l}\text { Mueller et al. } \\
2015\end{array}$ & SSD & $\begin{array}{l}\text { Integrated } \\
\text { Neurocogni- } \\
\text { tive Therapy } \\
\text { (INT) }\end{array}$ & B & 7 & $\begin{array}{l}\mathrm{EP}, \mathrm{ToM}, \mathrm{SP} \text {, } \\
\mathrm{SF} \text {, and neu- } \\
\text { rocognition }\end{array}$ & TAU & $140(73 / 67)$ & 64.2/74.7 & RCT & $\begin{array}{l}\text { INT: } 34.6(8.5) \\
\text { TAU: } 33.8(8.7)\end{array}$ & $\begin{array}{l}30 \text { sess. } / 45 \\
\text { h./15 wks. }\end{array}$ & $6-8$ & $\begin{array}{l}\text { - POFA (EP) } \\
\text { - Emotion } \\
\text { Recogniton } \\
\text { Question- } \\
\text { naire } \\
\text { (Emorec) } \\
\text { (EP) } \\
\text { - Schema } \\
\text { Component } \\
\text { Sequencing } \\
\text { Task-Revised } \\
\text { (SCST-R) } \\
\text { (SP) }\end{array}$ & $9 \mathrm{mos}$. & $\begin{array}{l}\text { Improvement } \\
\text { on all } \\
\text { outcome } \\
\text { measures. }\end{array}$ \\
\hline
\end{tabular}


TABLE 2

Continued

\begin{tabular}{|c|c|c|c|c|c|c|c|c|c|c|c|c|c|c|c|}
\hline Study & Population & $\begin{array}{l}\text { Name of } \\
\text { intervention }\end{array}$ & $\begin{array}{l}\text { Type of } \\
\text { intervention }\end{array}$ & $\begin{array}{l}\text { PEDro-P } \\
\text { score } \\
(0-10)\end{array}$ & $\begin{array}{l}\text { Intervention- } \\
\text { target(s) }\end{array}$ & $\begin{array}{l}\text { Control } \\
\text { group }\end{array}$ & $\begin{array}{l}\text { Sample size } \\
(\mathrm{Nt} / \mathrm{Nc})\end{array}$ & $\%$ Male & Design & Age of patients & $\begin{array}{l}\text { Duration/ } \\
\text { dose of } \\
\text { intervention }\end{array}$ & $\begin{array}{l}\text { Group } \\
\text { size }\end{array}$ & $\begin{array}{l}\text { Outcome } \\
\text { measures }\end{array}$ & $\begin{array}{l}\text { Follow- } \\
\text { up }\end{array}$ & Main findings \\
\hline $\begin{array}{l}\text { Neumann } \\
\text { et al., } 2014\end{array}$ & $A B I$ & $\begin{array}{l}\text { Stories } \\
\text { Intervention }\end{array}$ & $\mathrm{T}$ & 7 & EP & $\begin{array}{l}\text { Computer } \\
\text { games (NI) }\end{array}$ & $47(23 / 24)$ & 78/67 & RCT & $\begin{array}{l}\text { Stories:41.5 } \\
\text { (11.6) } \\
\text { Control: } 39.5 \\
(10.3)\end{array}$ & $\begin{array}{l}9 \text { sess./ } 9 \\
\text { h./3 wks. }\end{array}$ & 1 & $\begin{array}{l}\text { - DANVA 2-AF } \\
\text { (EP) } \\
\text { - EIST (EP) } \\
\text { - IRI (ToM) }\end{array}$ & $\begin{array}{l}3 \& 6 \\
\text { mos. }\end{array}$ & $\begin{array}{l}\text { No } \\
\text { improvement } \\
\text { on the } \\
\text { outcome } \\
\text { measures. }\end{array}$ \\
\hline $\begin{array}{l}\text { Pino et al., } \\
2015\end{array}$ & SSD & ETIT & C & 6 & EP and ToM & PST (NSCl) & $14(7 / 7)$ & $57.14 / 42.86$ & RCT & $\begin{array}{l}\text { ETIT: } 45 \\
(16.91) \\
\text { Control: } 42.25 \\
(8.20)\end{array}$ & $\begin{array}{l}24 \text { sess./ } 20 \\
\text { h./ } 12 \text { wks. }\end{array}$ & 7 & $\begin{array}{l}\text { - PSP (SF) } \\
\text { - Advanced } \\
\text { ToM Task } \\
\text { (ToM) } \\
\text { - Social } \\
\text { Situation Task } \\
\text { (SP) } \\
\text { - EAT (EP) } \\
\text { - RMET (EP) } \\
\text { - EQ (ToM) }\end{array}$ & - & $\begin{array}{l}\text { Improvement } \\
\text { on Advanced } \\
\text { ToM Task, } \\
\text { EAT, and PSP. }\end{array}$ \\
\hline $\begin{array}{l}\text { Radice- } \\
\text { Neumann } \\
\text { et al. } 2009\end{array}$ & $A B I$ & $\begin{array}{l}\text { Facial Affect } \\
\text { Recognition } \\
\text { Training } \\
\text { (FAR) }\end{array}$ & $\mathrm{T}$ & 4 & EP & $\begin{array}{l}\text { Stories of } \\
\text { Emotional } \\
\text { Inference } \\
\text { Training } \\
\text { (SCl) }\end{array}$ & 19 (10/9) & $82 / 33$ & RCT & $\begin{array}{l}\text { FAR: } 47 \text { (6.31) } \\
\text { SCI: } 38 \text { (14.37) }\end{array}$ & $\begin{array}{l}6-9 \text { sess./6- } \\
\text { 9h./2-3 } \\
\text { wks. }\end{array}$ & 1 & $\begin{array}{l}\text { - LEAS (EP) } \\
\text { - DANVA2-AF } \\
\text { (EP) } \\
\text { - DANVA2-AP } \\
\text { (EP) } \\
\text { - TASIT-1 (EP) } \\
\text { - BAFQ (SF) }\end{array}$ & 2 wks. & $\begin{array}{l}\text { Improvement } \\
\text { on } \\
\text { DANVA2-AF, } \\
\text { LEAS, and } \\
\text { BAFQ. }\end{array}$ \\
\hline $\begin{array}{l}\text { Roberts \& } \\
\text { Penn } 2009\end{array}$ & SSD & SCIT & c & 3 & EP and ToM & $\begin{array}{l}\text { E.g. } \\
\text { medication } \\
\text { manage- } \\
\text { ment, } \\
\text { psychother- } \\
\text { apy, } \\
\text { caseman- } \\
\text { agement, } \\
\text { family } \\
\text { education } \\
\text { and support } \\
\text { (TAU) }\end{array}$ & $25(14 / 11)$ & $55 / 64$ & $\begin{array}{l}\text { Quasi- } \\
\text { experiment }\end{array}$ & $\begin{array}{l}\text { SCIT: } 36.8 \\
(12.3) \\
\text { TAU: } 41.4 \\
(12.3)\end{array}$ & 20 wks. & 4-11 & $\begin{array}{l}\text { - FEIT (EP) } \\
\text { - BLERT (EP) } \\
\text { - HT (ToM) } \\
\text { - TASIT- } \\
\text { abbreviated } \\
\text { (ToM) } \\
\text { - SSPA (SF) }\end{array}$ & - & $\begin{array}{l}\text { Improvement } \\
\text { on FEIT and } \\
\text { SSPA. }\end{array}$ \\
\hline
\end{tabular}


TABLE 2

Continued

\begin{tabular}{|c|c|c|c|c|c|c|c|c|c|c|c|c|c|c|c|}
\hline Study & Population & $\begin{array}{l}\text { Name of } \\
\text { intervention }\end{array}$ & $\begin{array}{l}\text { Type of } \\
\text { intervention }\end{array}$ & $\begin{array}{l}\text { PEDro-P } \\
\text { score } \\
(0-10)\end{array}$ & $\begin{array}{l}\text { Intervention- } \\
\text { target(s) }\end{array}$ & $\begin{array}{l}\text { Control } \\
\text { group }\end{array}$ & $\begin{array}{l}\text { Sample size } \\
(\mathrm{Nt} / \mathrm{Nc})\end{array}$ & $\begin{array}{l}\% \\
\text { Male }\end{array}$ & Design & Age of patients & $\begin{array}{l}\text { Duration/ } \\
\text { dose of } \\
\text { intervention }\end{array}$ & $\begin{array}{l}\text { Group } \\
\text { size }\end{array}$ & $\begin{array}{l}\text { Outcome } \\
\text { measures }\end{array}$ & $\begin{array}{l}\text { Follow- } \\
\text { up }\end{array}$ & Main findings \\
\hline $\begin{array}{l}\text { Roberts et al. } \\
2014\end{array}$ & SSD & SCIT & C & 5 & EP and ToM & $\begin{array}{l}\text { E.g. } \\
\text { pharmaco- } \\
\text { therapy, } \\
\text { case- } \\
\text { management, } \\
\text { psychother- } \\
\text { apy } \\
\text { (TAU) }\end{array}$ & $63(32 / 31)$ & $67 / 67$ & RCT & $\begin{array}{l}\text { SCIT: } 40.0 \\
\text { (12.2) } \\
\text { TAU: } 39.4 \\
(10.8)\end{array}$ & $\begin{array}{l}20-24 \\
\text { sess./20- } \\
24 \mathrm{~h} . / 20-24 \\
\text { wks. }\end{array}$ & $4-8$ & $\begin{array}{l}\text { - FEIT (EP) } \\
\text { - FEDT (EP) } \\
\text { - ERT-40 } \\
\text { (EP) } \\
\text { - HT (ToM) } \\
\text { - TASIT } \\
\text { (ToM) } \\
\text { - OSCARS } \\
\text { (SF) } \\
\text { - SSPA (SF) } \\
\text { - GSFS (SF) }\end{array}$ & $3 \mathrm{mos}$. & $\begin{array}{l}\text { Improvement } \\
\text { on SSPA. }\end{array}$ \\
\hline $\begin{array}{l}\text { Rocha \& } \\
\text { Queirós } 2013\end{array}$ & SSD & $\begin{array}{l}\text { Metacognitive } \\
\text { and Social } \\
\text { Cognitive } \\
\text { Training } \\
\text { (MCST) }\end{array}$ & C & 4 & $\begin{array}{l}\text { EP, ToM, and } \\
\text { SP }\end{array}$ & $\begin{array}{l}\text { E.g. } \\
\text { medication, } \\
\text { psychoso- } \\
\text { cial services, } \\
\text { life and } \\
\text { social skills } \\
\text { training, } \\
\text { psycho- } \\
\text { education, } \\
\text { stress- } \\
\text { management, } \\
\text { psychother- } \\
\text { apy, } \\
\text { family- } \\
\text { education } \\
\text { (TAU) }\end{array}$ & 35 (19/16) & $84 / 94$ & $\begin{array}{l}\text { Quasi- } \\
\text { experiment }\end{array}$ & $\begin{array}{l}\text { MCST: } 38.63 \\
(8.88) \\
\text { TAU: } 35.94 \\
(8.69)\end{array}$ & $\begin{array}{l}18 \text { sess./10 } \\
\text { wks. }\end{array}$ & Unknow & $\begin{array}{l}\text { - FEIT (EP) } \\
\text { n- MSCEIT } \\
\text { (EP) } \\
\text { - HT (ToM) } \\
\text { - SPS (SP) } \\
\text {-LSP (SF) }\end{array}$ & - & $\begin{array}{l}\text { Improvement } \\
\text { on LSP. }\end{array}$ \\
\hline $\begin{array}{l}\text { Roncone } \\
\text { et al. } 2004\end{array}$ & SSD & $\begin{array}{l}\text { Instrumental } \\
\text { Enrichment } \\
\text { Programme } \\
\text { (IEP) }\end{array}$ & C & 5 & $\begin{array}{l}\text { EP, ToM, and } \\
\text { SF }\end{array}$ & $\begin{array}{l}\text { E.g. } \\
\text { medication, } \\
\text { support, } \\
\text { psychother- } \\
\text { apy } \\
\text { (TAU) }\end{array}$ & $20(10 / 10)$ & $60 / 70$ & RCT & $\begin{array}{l}\text { IEP: } 33.9 \\
\text { TAU: } 33.5\end{array}$ & $\begin{array}{l}22 \text { sess./22 } \\
\text { h./22 wks. }\end{array}$ & 10 & $\begin{array}{l}\text { - False belief } \\
\text { stories (ToM) } \\
\text { - ERT (EP) }\end{array}$ & - & $\begin{array}{l}\text { Improvement } \\
\text { on ToM and } \\
\text { ERT Sadness } \\
\text { and Fear. }\end{array}$ \\
\hline
\end{tabular}


TABLE 2

Continued

\begin{tabular}{|c|c|c|c|c|c|c|c|c|c|c|c|c|c|c|c|}
\hline Study & Population & $\begin{array}{l}\text { Name of } \\
\text { intervention }\end{array}$ & $\begin{array}{l}\text { Type of } \\
\text { intervention }\end{array}$ & $\begin{array}{l}\text { PEDro-P } \\
\text { score } \\
(0-10)\end{array}$ & $\begin{array}{l}\text { Intervention- } \\
\text { target(s) }\end{array}$ & $\begin{array}{l}\text { Control } \\
\text { group }\end{array}$ & $\begin{array}{l}\text { Sample size } \\
(\mathrm{Nt} / \mathrm{Nc})\end{array}$ & $\%$ Male & Design & Age of patients & $\begin{array}{l}\text { Duration/ } \\
\text { dose of } \\
\text { intervention }\end{array}$ & $\begin{array}{l}\text { Group } \\
\text { size }\end{array}$ & $\begin{array}{l}\text { Outcome } \\
\text { measures }\end{array}$ & $\begin{array}{l}\text { Follow- } \\
\text { up }\end{array}$ & Main findings \\
\hline $\begin{array}{l}\text { Rus-Calafell } \\
\text { et al. } 2013\end{array}$ & SSD & SST & B & 5 & $\begin{array}{l}\text { ToM, SP, SF, } \\
\text { and social } \\
\text { skills }\end{array}$ & $\begin{array}{l}\text { E.g. case- } \\
\text { management, } \\
\text { psychother- } \\
\text { apy, } \\
\text { medication } \\
\text { adherence, } \\
\text { family } \\
\text { support, } \\
\text { leisure } \\
\text { engagement } \\
\text { (TAU) }\end{array}$ & 31 (13/18) & $77 / 83$ & RCT & $\begin{array}{l}\text { SST } 37.54 \\
(8.05) \\
\text { TAU } 42.39 \\
(8.1)\end{array}$ & 16 sess. & 9 & $\begin{array}{l}\text { - Al } \\
\text { behaviour } \\
\text { (SF) } \\
\text { - SSIT (SF) } \\
\text { - SFS (SF) }\end{array}$ & - & $\begin{array}{l}\text { Improvement } \\
\text { on SFS. }\end{array}$ \\
\hline $\begin{array}{l}\text { Russell et al. } \\
2008\end{array}$ & SSD & $\begin{array}{l}\text { Micro } \\
\text { Expression } \\
\text { Training } \\
\text { Tool (METT) }\end{array}$ & $\mathrm{T}$ & 5 & EP & $\begin{array}{l}\text { Repeated } \\
\text { exposure } \\
\text { (NI) }\end{array}$ & $40(26 / 14)$ & $65 / 71$ & RCT & $\begin{array}{l}\text { MET: } 40 \text { (10) } \\
\text { NI: } 44 \text { (9) }\end{array}$ & 1 sess. $/ 3 \mathrm{~h}$. & 1 & - JCFEE (EP) & 1 wk. & $\begin{array}{l}\text { Improvement } \\
\text { on JCFEE. }\end{array}$ \\
\hline $\begin{array}{l}\text { Sachs et al. } \\
2012\end{array}$ & SSD & TAR & $\mathrm{T}$ & 5 & EP & TAU & $38(20 / 18)$ & $60 / 40$ & RCT & $\begin{array}{l}\text { TAR: } 27.20 \\
\text { (7.17) } \\
\text { TAU: } 31.72 \\
(9.35)\end{array}$ & $\begin{array}{l}12 \text { sess. } 6 \\
\text { wks. }\end{array}$ & Unknown & $\begin{array}{l}\text { - VERT-K } \\
\text { (EP) }\end{array}$ & - & $\begin{array}{l}\text { Improvement } \\
\text { on VERT-K. }\end{array}$ \\
\hline $\begin{array}{l}\text { Tas et al. } \\
2012\end{array}$ & SSD & $\begin{array}{l}\text { Family- } \\
\text { Assisted } \\
\text { SCIT } \\
\text { (F-SCIT) }\end{array}$ & C & 6 & EP and ToM & $\begin{array}{l}\text { Social } \\
\text { stimulation } \\
\text { (NI) }\end{array}$ & $45(19 / 26)$ & $58 / 46$ & RCT & $\begin{array}{l}\text { F-SCIT: } 33.32 \\
(11.57) \\
\text { NI: } 34.62 \\
(10.06)\end{array}$ & $\begin{array}{l}14 \text { sess./19 } \\
\text { h./20 wks. } \\
\text { (group) } \\
\text { combined } \\
\text { with one } \\
\text { individual } \\
\text { sess. }\end{array}$ & $9-10$ & $\begin{array}{l}\text { - FEIT (EP) } \\
\text { - FEDT (EP) } \\
\text { - HT (ToM) } \\
\text { - RMET (EP) } \\
\text { - UOT (ToM) } \\
\text {-SFS (SF) }\end{array}$ & - & $\begin{array}{l}\text { Improvement } \\
\text { on HT, FEIT, } \\
\text { FEDT, UOT, } \\
\text { and SFS } \\
\text { social } \\
\text { withdrawal. }\end{array}$ \\
\hline $\begin{array}{l}\text { Taylor et al. } \\
2015\end{array}$ & SSD & $\begin{array}{l}\text { SCIT- } \\
\text { adapted } \\
\text { (a) }\end{array}$ & C & 5 & EP and ToM & TAU & $27(16 / 11)$ & - & RCT & $\begin{array}{l}\text { SCIT-a: } 40.7 \\
(10.3) \\
\text { Control: } 39.2 \\
(10.6)\end{array}$ & $\begin{array}{l}16 \text { sess./12 } \\
\text { h./8 wks. }\end{array}$ & $?$ & $\begin{array}{l}\text { - FEIT (EP) } \\
\text { - HT (ToM) }\end{array}$ & - & $\begin{array}{l}\text { Improvement } \\
\text { on FEIT. }\end{array}$ \\
\hline
\end{tabular}


TABLE 2

Continued

\begin{tabular}{|c|c|c|c|c|c|c|c|c|c|c|c|c|c|c|c|}
\hline Study & Population & $\begin{array}{l}\text { Name of } \\
\text { intervention }\end{array}$ & $\begin{array}{l}\text { Type of } \\
\text { intervention }\end{array}$ & $\begin{array}{l}\text { PEDro-P } \\
\text { score } \\
(0-10)\end{array}$ & $\begin{array}{l}\text { Intervention- } \\
\text { target(s) }\end{array}$ & $\begin{array}{l}\text { Control } \\
\text { group }\end{array}$ & $\begin{array}{l}\text { Sample size } \\
(\mathrm{Nt} / \mathrm{Nc})\end{array}$ & $\%$ Male & Design & Age of patients & $\begin{array}{l}\text { Duration/ } \\
\text { dose of } \\
\text { intervention }\end{array}$ & $\begin{array}{l}\text { Group } \\
\text { size }\end{array}$ & $\begin{array}{l}\text { Outcome } \\
\text { measures }\end{array}$ & $\begin{array}{l}\text { Follow- } \\
\text { up }\end{array}$ & Main findings \\
\hline $\begin{array}{l}\text { Turner- } \\
\text { Brown et al. } \\
2008\end{array}$ & ASD & $\begin{array}{l}\text { SCIT- } \\
\text { Autism } \\
\text { (SCIT-A) }\end{array}$ & c & 2 & $\begin{array}{l}\mathrm{EP}, \mathrm{ToM} \text {, and } \\
\text { SP }\end{array}$ & TAU & $11(6 / 5)$ & $83 / 100$ & $\begin{array}{l}\text { Quasi- } \\
\text { experiment }\end{array}$ & $\begin{array}{l}\text { SCIT-A: } 42.5 \\
\text { (12.3) } \\
\text { TAU: } 28.8(1.0)\end{array}$ & $\begin{array}{l}18 \text { sess./15 } \\
\text { h./18 wks. }\end{array}$ & $5-6$ & $\begin{array}{l}\text { - FEIT (EP) } \\
\text { - HT (ToM) } \\
\text { - SCSQ (SF) } \\
\text { - SSPA (SF) }\end{array}$ & - & $\begin{array}{l}\text { Improvement } \\
\text { on HT. }\end{array}$ \\
\hline $\begin{array}{l}\text { Veltro et al. } \\
2011\end{array}$ & SSD & $\begin{array}{l}\text { Cognitive- } \\
\text { Emotional } \\
\text { Rehabilita- } \\
\text { tion } \\
\text { (REC) }\end{array}$ & c & 7 & EP and ToM & $\begin{array}{l}\text { Problem } \\
\text { solving } \\
\text { training } \\
\text { (NSCl) }\end{array}$ & $24(12 / 12)$ & - & RCT & $\begin{array}{l}\text { REC: } 38.8(6.3) \\
\text { NSCl: } 37.7 \\
(11.16)\end{array}$ & $\begin{array}{l}+/-24 \\
\text { sess./33 } \\
\text { h./24 wks. }\end{array}$ & $8-12$ & $\begin{array}{l}\text { - PSP (SF) } \\
\text { - Advanced } \\
\text { ToM Scale } \\
\text { (ToM) } \\
\text { - EAT (EP) }\end{array}$ & - & $\begin{array}{l}\text { Improvement } \\
\text { on Advanced } \\
\text { ToM Scale } \\
\text { and EAT. }\end{array}$ \\
\hline $\begin{array}{l}\text { Wölwer et al. } \\
2005\end{array}$ & SSD & TAR & $\mathrm{T}$ & 4 & EP & $\begin{array}{l}\text { E.g. } \\
\text { medication, } \\
\text { psycho- } \\
\text { educational } \\
\text { therapy } \\
(\mathrm{TAU})\end{array}$ & $53(28 / 25)$ & $89 / 84$ & RCT & $\begin{array}{l}\text { TAR } 31.5(6.9) \\
\text { TAU } 35.2 \\
(11.1)\end{array}$ & $\begin{array}{l}12 \text { sess./9 } \\
\text { h./6 wks. }\end{array}$ & 2 & - POFA (EP) & - & $\begin{array}{l}\text { Improvement } \\
\text { on POFA. }\end{array}$ \\
\hline $\begin{array}{l}\text { Wölwer \& } \\
\text { Frommann } \\
2011\end{array}$ & SSD & TAR & $\mathrm{T}$ & 6 & EP & $\begin{array}{l}\text { Cognitive } \\
\text { Remediation } \\
\text { Therapy } \\
\text { (NSCl) }\end{array}$ & $38(20 / 18)$ & 68 & RCT & $\begin{array}{l}\text { TAR/NSCl: } \\
36.7 \text { (13.1) }\end{array}$ & $\begin{array}{l}12 \\
\text { sess./+/- } \\
10.5 \text { h./6 } \\
\text { wks. }\end{array}$ & 2 & $\begin{array}{l}\text { - GVEESS } \\
\text { (EP) } \\
\text { - PST (ToM) } \\
\text { - RPT (SF) }\end{array}$ & - & $\begin{array}{l}\text { Improvement } \\
\text { on all } \\
\text { measures. }\end{array}$ \\
\hline
\end{tabular}

Note: SSD = schizophrenia spectrum disorders; $A S D=$ autism spectrum disorders; $A B I=$ acquired brain injury; $B=$ broad-based intervention; $C=C$ Comprehensive intervention; $T=$

Targeted intervention; TAU = treatment as usual; $\mathrm{NI}=$ no intervention; $\mathrm{NSCl}=$ no social cognitive intervention; $\mathrm{SCl}=$ other social cognitive intervention; $\mathrm{Nt}=\mathrm{N}$ treatment group; $\mathrm{Nc}=$ $N$ control group; $E P=$ emotion perception, ToM = theory of mind; SP = social perception, $S F=$ social functioning, POFA = Pictures of Facial Affect; PST = ToM Picture Sequencing Task; FENT = Facial Expression Naming Task; FEMT = Facial Expression Matching Task; TASIT = The Awareness of Social Inference Test; FES/DT = Facial Expression Same/Different Task; FEIT = Face Emotion Identification Test; FEDT = Face Emotion Discrimination Test; SPS = Social Perception Scale; HT = Hinting Task; SFS = Social Functioning Scale, social withdrawal \& interpersonal behavior; MSCEIT = Mayer-Salovey-Caruso Emotional Intelligence Test, managing emotions; CFER = Computerised test of Facial Emotion Recognition; CAM = Cambridge Mindreading Face-Voice Battery; RMET = Reading the Mind in the Eyes Task; RMVT = Reading the Mind in the Voice Task; RMFT = Reading the Mind in the Film Task; PONS = Half-Profile of Nonverbal Sensitivity; ER-40: Emotion Recognition 40; PSP = Personal and Social Performance Scale, personal and social relationships; EAT = Emotion

Attribution Task; EQ = Empathy Questionnaire; SPSS = Social Performance Survey Schedule; BRISS-R PDBS/PCSS = Behavioural Referenced Rating System of Intermediate Social Skills - Revised Partner Directed Behaviour Scale/Personal Conversational Style Scale; LEAS = Levels of Emotional Awareness Scale; DANVA2 AF/AP = Diagnostic Assessment of Nonverbal Affect 2- Adult Faces/Adult Paralanguage; BAFQ = Brock Adaptive Functioning Questionnaire; SSPA = Social Skills Performance Assessment; ERT-40- Emotion Recognition Test-40 faces edition; OSCARS = Observable Social Cognition: A Rating Scale; GSFS = Global Social Functioning Scale; LSP = Life Skills Profile, social contact; ERT = Emotion Recognition Task; Al: Assertion Inventory; SSIT = Simulated Social Interaction Test; JCFEE = Japanese and Caucasian Facial Expression Emotion; VERT-K = Vienna Emotion Recognition Task; UOT = Unexpected Outcomes Test; SCSQ = Social Communication Skills Questionnaire; GVEESS = Geneva Vocal Emotion Expression Stimulus; RPT = Role Play Task; EIST = Emotional Inference from Stories Test; IRI = Interpersonal Reactivity Index, perspective taking and empathic concern. 
controlled trial design. In eight studies, a quasiexperimental design was used (Bechi et al., 2012; Combs et al., 2007; Golan \& Baron-Cohen, 2006, exp. 2; Lahera et al., 2013; McDonald et al., 2008; Roberts \& Penn, 2009; Rocha \& Queirós, 2013; Turner-Brown, Perry, Dichter, Bodfish \& Penn, 2008).

The studies included in total 1,508 participants (794 patients in intervention groups and 714 in control groups), involving patients with SSD (schizophrenia, schizoaffective disorder, schizoaffective psychosis, schizophreniform disorder, delusional disorder, psychosis not otherwise specified, $k=30$ ), ABI (mainly including patients with traumatic brain injury, $k=6$ ), and ASD (Autism, Asperger syndrome, highfunctioning autism, $k=4$ ). One study included a population of patients with bipolar or schizoaffective disorder. The mean age of patients in the included studies ranged from 24.4 (Mazza et al., 2010) to 51.0 (Horan et al., 2011). In almost all studies, the number of men was higher than women. Sample sizes varied widely from 10 patients (Bölte et al., 2006; Bornhofen \& McDonald, 2008b) to 140 (Mueller, Schmidt \& Roder, 2015).

Table 2 shows the consensus ratings on the PEDro-P scale. Most studies $(k=16)$ received a score of 5 out of 10 points, which was also the average score over all 41 studies. The study with the lowest score (a PEDro-P total score of 2 points) concerned a pilot study (Turner-Brown et al., 2008), explaining why its methodological quality was considered to be lower than that of other studies. Four studies received the highest score of 7 out of 10 points (McDonald et al., 2013; Mueller et al., 2015; Neumann et al., 2014; Veltro et al., 2011). It should be noted that these studies could never receive the maximum number of 10 points, because blinding of the therapists who administer the treatment is not possible in most psychological intervention studies (item 6 of the PEDro-P scale). Therefore, the studies could only receive a maximum score of 9 points. Moreover, only two studies met the criterion of blinding all participants (Golan et al., 2006, experiment 1; Golan et al., 2006, experiment 2).

All interventions could be classified as broadbased $(k=10)$, comprehensive $(k=16)$, or targeted interventions $(k=15)$. Some interventions were evaluated by multiple studies: SCIT (Combs et al., 2007; Lahera et al., 2013; Roberts \& Penn, 2009; Roberts et al., 2014; Tas, Danaci, Cubukcuoglu \& Brüne, 2012; Taylor et al., 2015; Turner-Brown et al., 2008), CET (Eack et al., 2009; Hogarty et al., 2004), Mind Reading (Golan \& Baron-Cohen, 2006; Lindenmayer et al., 2013), TAR (Sachs et al., 2012, Wölwer et al., 2005; Wölwer \& Frommann, 2011), Social Cognitive Skills Training (SCST, Horan et al., 2009, 2011), SST (McDonald et al., 2008; Rus-Calafell, Gutiérrez-Maldonado, OrtegaBravo, Ribas-Sabaté \& Caqueo-Urízar, 2013), Emotion and ToM Imitation Training (ETIT: Mazza et al., 2010; Pino, Pettinelli, Clementi, Gianfelice \& Mazza, 2015), Theory of Mind Intervention (ToMI: Bechi et al., 2013, 2015), and the Social Cognition Training Program (SCTP: GilSanz et al., 2009, 2014).

Overall, emotion perception, specifically or amongst other SC subdomains, was the most frequently investigated treatment target $(k=34)$. Twenty-five studies aimed to ameliorate ToM, 13 focussed on social perception, and six studies aimed to improve social functioning. No intervention studies for remediating alexithymic problems could be included, because the studies focussed on non-neuropsychiatric participants (for instance, somatic patients), used alexithymia as a moderator of treatment success rather than as an outcome measure, or did not meet the other inclusion criteria (see e.g., Cameron et al., 2014; Samur et al, 2013; Vanheule, Verhaeghe \& Desmet, 2011 for reviews on alexithymia interventions). Targeted interventions predominantly focussed on training emotion perception $(k=13)$, comprehensive interventions mostly trained emotion perception $(k=16)$ combined with ToM $(k=15)$, and broad-based interventions almost exclusively combined neurocognitive training $(k=8)$ or social skills training $(k=2)$ with ToM treatment $(k=9)$.

Intervention duration varied widely from a single session in one week (Combs et al., 2008) to a combination of 56 sessions SC training with 75 sessions neurocognitive training for 104 weeks (Hogarty et al., 2004). The intensity of treatments, expressed in treatment hours, ranged from 2 hours (Kayser, Sarfati, Besche \& Hardy-Baylé, 2007) to 159 hours (Hogarty et al., 2004). Session duration was also variable and commonly ranged between 45 (Wölwer et al., 2005) and 180 minutes (Garcia, Fuentes, Ruiz, Gallach, \& Roder, 2003; McDonald et al., 2008; Russel et al., 2008) per session. Group size ranged between an individual setting (Combs et al., 2008; Golan \& Baron-Cohen, 2006; Radice-Neumann, Zupan, Tomita \& Willer, 2009; Russell, Green, Simpson \& Coltheart, 2008) and groups of up to 12 participants (Lahera et al., 2013; Veltro et al., 2011). Intervention and session duration, intensity, and group size were not specified in some studies (Bölte et al., 2006; Eack et al., 2009; Gil Sanz et al., 2009; Lindenmayer et al., 2013; Mazza et al., 2010; Roberts \& Penn, 2009; Rocha \& Queirós, 2013; Sachs et al., 2012; Taylor et al., 
2015). In general, targeted interventions were the least intensive training type, in terms of treatment duration and intensity. Broad-based interventions were most intensive.

The activities of the patients in the control groups were diverse. Sixteen studies included a treatment as usual condition (e.g., illness management, pharmacotherapy), 11 studies included no intervention (e.g., waiting list, deferred treatment) as control group, 11 studies used an intervention that was not SC in nature (e.g., neurocognitive intervention, problem solving skills training), two studies used another SC intervention, and for one study the nature of the control group was not specified.

A variety of SC outcome measures was used in the selected studies (see Table 1 for an overview). For measuring emotion perception, the following tests were administered most frequently: the Face Emotion Identification Test (FEIT, Kerr \& Neale, 1993, $k=12$ ), the Face Emotion Discrimination Test (FEDT, Kerr \& Neale, 1993, $k=5$ ), the Reading the Mind in the Eyes Task Revised (Baron-Cohen, Wheelwright, Hill, Raste \& Plumb, 2001, $k=5$ ), and The Awareness of Social Inference Test-Part 1 (TASIT-1, McDonald, Flanagan, Rollins \& Kinch, 2003; McDonald et al., 2006, $k=5$ ). To assess social perception, the Social Perception Scale (SPS, Garcia et al. 2003, $k=4$ ) and Half-Profile of Non-verbal Sensitivity (PONS, Ambady, Hallhan, \& Rosenthal, 1995; Rosenthal, Hall, DiMatteo, Rogers \& Archer, 1979, $k=2$ ) were used most frequently. Most commonly used measures of ToM included the Hinting Task (Corcoran, Mercer \& Firth, 1995, $k=10$ ), TASIT-Parts 2 and 3 (McDonald et al., 2003, 2006, $k=6$ ), and the Reading the Mind in the Eyes Task Revised (Baron-Cohen et al., 2001, $k=5$ ). Regarding social functioning, the subtest managing emotions of the Mayer-Salovey-Caruso Emotional Intelligence Test (MSCEIT, Mayer, Salovey \& Caruso, 2002; Mayer, Salovey, Caruso \& Sitarenios, 2003, $k=4$ ) was used most commonly, but also the Social Functioning Scale (SFS, Birchwood, Smith, Cochrane, Wetton \& Copestake, 1990, $k=4$ ), the Personal and Social Performance Scale (PSP, Hsieh et al., 2011; Morosini, Magliano, Brambilla, Ugolini \& Piolo, 2000, $k=3$ ), and the Social Skills Performance Assessment (SSPA, Patterson, Moscona, McKibbin, Davidson \& Jeste, $2001, k=3$ ) were administered frequently.

Ten studies performed a follow-up assessment after a non-treatment period (Bornhofen \& McDonald, 2008a; Bornhofen \& McDonald, 2008b; Combs et al., 2008; Hogarty et al., 2004; Hogarty, Greenwald, \& Eack, 2006; McDonald et al., 2013; Mueller et al., 2015; Neumann, Bab- bage, Zupan \& Willer, 2014; Radice-Neumann et al., 2009; Roberts et al., 2014; Russell et al., 2008). Follow-up test intervals were highly variable and ranged from one week post-treatment (Combs et al., 2008; Russell et al., 2008) to three years post-treatment (Hogarty et al., 2004, data were published in Hogarty et al., 2006). It is notable that seven of the ten follow-up studies concerned targeted interventions, one comprehensive intervention, and two broad-based interventions. Overall, eight studies found maintenance effects at follow-up (mostly compared with baseline) at one or more outcome measures.

\section{Overall Effects of the SC Interventions}

First, we present the overall results of the metaanalyses, investigating the effects of SC interventions on SC, followed by the specific effects on the four SC subdomains. Next, we describe the effects of the three different treatment types on SC. Finally, the intervention effects for the three patient groups are reported. Tables 3 and 4 provide an overview of all the effects and test statistics of the meta-analyses.

The main meta-analysis investigated the effect of the interventions on SC in general. The metaanalysis showed evidence of a moderate-to-large intervention effect $(\mathrm{SMD}=.71,95 \% \mathrm{CI}=.56-$ $.86, Z=9.15, p<.001$ ) (Figure 2). Regarding the SC subdomains, the meta-analysis showed moderate-to-large intervention effects on emotion perception $(\mathrm{SMD}=.79,95 \% \mathrm{CI}=.60-.98$, $Z=8.07, p<.001)$. Moderate effects were found on ToM, social perception, and social functioning $\left(\mathrm{SMD}_{\mathrm{ToM}}=.64,95 \% \mathrm{CI}=.40-.89, Z=5.18\right.$, $p<.001 ; \mathrm{SMD}_{\text {social perception }}=.52,95 \% \mathrm{CI}=-.01-$ $1.06, Z=1.93, p=.05 ; \mathrm{SMD}_{\text {social functioning }}=.61$, $95 \% \mathrm{CI}=.42-.81, Z=6.30, p<.001)$.

\section{Effects of Targeted Interventions}

The meta-analysis resulted in a large effect on $\mathrm{SC}(\mathrm{SMD}=.89,95 \% \mathrm{CI}=.58-1.20$, $Z=5.67, p<.001$ ) (Figure 3). Regarding the SC subdomains, targeted interventions had large effects on emotion perception, social perception, and ToM $\quad\left(\mathrm{SMD}_{\text {emotion perception }}=.83,95 \% \mathrm{CI}=.50\right.$ $1.16, Z=4.92, p<.001 ; \mathrm{SMD}_{\text {social perception }}=1.33$, 95\% $\mathrm{CI}=.34-2.33, \quad \mathrm{Z}=2.63, \quad p<.01$; $\mathrm{SMD}_{\mathrm{ToM}}=.86,95 \% \mathrm{CI}=.29-1.43, Z=2.94, p<$ .01). A borderline significant, moderate effect was found on social functioning $(\mathrm{SMD}=.52,95 \%$ $\mathrm{CI}=-.01-1.05, Z=1.93, p=.05)$. 
TABLE 3

Sample Sizes, Standardised Mean Differences, Test Statistics, and Fail-safe N of the Included Social Cognitive Intervention Studies Organised by Intervention Type and Outcome Domain

\begin{tabular}{|c|c|c|c|c|c|c|}
\hline & $k$ & $N(\mathrm{Nt} / \mathrm{Nc})$ & $\operatorname{SMD}(\mathrm{CI})$ & $Z$ & $\chi^{2}$ & $N_{\mathrm{fs}}$ \\
\hline \multicolumn{7}{|l|}{ All interventions } \\
\hline Social cognition & 41 & 1508 (794/714) & $0.71(0.56-0.86)$ & $9.15^{* * *}$ & $74.20^{* * *}$ & 2336 \\
\hline Emotion perception & 34 & $1191(617 / 574)$ & $0.79(0.60-0.98)$ & $8.07^{* * *}$ & $75.72 * * *$ & 1783 \\
\hline Social perception & 8 & $315(165 / 150)$ & $0.52(-0.01-1.06)$ & $1.92^{*}$ & $29.13^{* * *}$ & 34 \\
\hline Theory of Mind & 27 & $853(445 / 408)$ & 0.64 (0.40-0.89) & $5.18^{* * *}$ & $71.56^{* * *}$ & 667 \\
\hline Social functioning & 17 & $646(335 / 311)$ & $0.61(0.42-0.80)$ & $6.30^{* * *}$ & 21.01 & 312 \\
\hline \multicolumn{7}{|c|}{ Targeted interventions } \\
\hline Social cognition & 15 & $427(223 / 204)$ & $0.89(0.58-1.20)$ & $5.67^{* * *}$ & $28.83^{*}$ & 360 \\
\hline Emotion perception & 13 & 393 (204/189) & $0.83(0.50-1.16)$ & $4.92^{* * *}$ & $26.76^{* *}$ & 257 \\
\hline Social perception & 1 & $20(11 / 9)$ & $1.33(0.34-2.33)$ & $2.63^{* *}$ & - & 2 \\
\hline Theory of Mind & 7 & $187(93 / 94)$ & $0.86(0.29-1.43)$ & $2.94^{* *}$ & $18.06 * *$ & 38 \\
\hline Social functioning & 2 & $57(30 / 27)$ & $0.52(-0.01-1.05)$ & $1.93^{*}$ & 0.70 & 0 \\
\hline \multicolumn{7}{|c|}{ Comprehensive interventions } \\
\hline Social cognition & 16 & $484(247 / 237)$ & $0.61(0.35-0.87)$ & $4.65^{* * *}$ & $26.84^{*}$ & 217 \\
\hline Emotion perception & 16 & $484(247 / 237)$ & $0.83(0.52-1.15)$ & $5.17^{* * *}$ & $38.51^{* * *}$ & 367 \\
\hline Social perception & 6 & $155(81 / 74)$ & $0.46(-0.31-1.24)$ & 1.17 & $25.44^{* * *}$ & 8 \\
\hline Theory of Mind & 15 & $470(240 / 230)$ & $0.58(0.21-0.94)$ & $3.10^{* *}$ & $47.74^{* * *}$ & 182 \\
\hline Social functioning & 10 & $302(153 / 149)$ & $0.56(0.27-0.84)$ & $3.86^{* * *}$ & 12.26 & 72 \\
\hline \multicolumn{7}{|c|}{ Broad-based interventions } \\
\hline Social cognition & 10 & $597(324 / 273)$ & $0.65(0.43-0.87)$ & $5.81^{* * *}$ & 14.01 & 191 \\
\hline Emotion perception & 5 & $314(166 / 148)$ & $0.63(0.28-0.98)$ & $3.50^{* * *}$ & 8.18 & 39 \\
\hline Social perception & 1 & $140(73 / 67)$ & $0.33(-0.01-0.66)$ & 1.91 & - & 1 \\
\hline Theory of Mind & 5 & $196(112 / 84)$ & $0.64(0.34-0.93)$ & $4.26 * * *$ & 3.63 & 28 \\
\hline Social functioning & 5 & $287(152 / 135)$ & $0.74(0.40-1.08)$ & $4.25^{* * *}$ & 7.04 & 56 \\
\hline
\end{tabular}

Note: $k$, number of studies; $N$, number of patients; $\mathrm{Nt}$, number of patients in treatment group; $\mathrm{Nc}$, number of patients in control group; $Z$, significance test; $\chi^{2}$, heterogeneity statistic; Nfs, number of null findings needed for a non-significant combined effect size calculated by the method of Rosenthal (1979).

$* p \leq .05,{ }^{* *} p<.01,{ }^{* * *} p<.001$.

\section{Effects of Comprehensive Interventions}

The meta-analysis resulted in a moderate effect size on $\mathrm{SC}(\mathrm{SMD}=.61,95 \% \mathrm{CI}=.35-.87$, $Z=4.65, p<.001$ ) (Figure 4). Regarding the SC subdomains, comprehensive interventions had a large effect on emotion perception $(\mathrm{SMD}=.81$, $95 \% \mathrm{CI}=.52-1.15, Z=5.17, p<.001)$. For ToM and social functioning, moderate effects were found $\left(\mathrm{SMD}_{\mathrm{ToM}}=.58,95 \% \mathrm{CI}=.21-.94\right.$, $Z=3.10, p<.01 ; \mathrm{SMD}_{\text {social functioning }}=.56,95 \%$ $\mathrm{CI}=.27-.84, \mathrm{Z}=3.86, p<.001)$. The small effect on social perception was not significant $(\mathrm{SMD}=.46, \quad 95 \% \quad \mathrm{CI}=-.31-1.24, \quad \mathrm{Z}=1.17$, $p=.24)$.

\section{Effects of Broad-Based Interventions}

An overall moderate effect size on SC was found for the broad-based interventions $(\mathrm{SMD}=.65$,
$95 \% \mathrm{CI}=.43-.87, Z=5.81, p<.001$ ) (Figure 5). Regarding the four SC subdomains, broad-based interventions resulted in a moderate-to-large effect on social functioning $(\mathrm{SMD}=.74,95 \%$ $\mathrm{CI}=.40-1.08, Z=4.25, p<.001)$. Moderate effects were found on emotion perception and $\mathrm{ToM}\left(\mathrm{SMD}_{\text {emotion perception }}=.63,95 \% \mathrm{CI}=.28-.98\right.$, $Z=3.50, p<.001 ; \mathrm{SMD}_{\mathrm{ToM}}=.64,95 \%=.34$ $.93, Z=4.26, p<.001)$. Only a small, but non-significant effect was found for social perception, based on only one broad-based intervention $(\mathrm{SMD}=.33, \quad 95 \% \quad \mathrm{CI}=-.01-.66, \quad Z=1.91$, $p=.06)$.

\section{Effects of the SC Interventions Per Patient Group}

Table 4 shows all effects sizes and test statistics for the three patient groups. In patients with 
TABLE 4

Sample Sizes, Standardised Mean Differences, Test Statistics, and Fail-safe $N$ of the Included Social Cognitive Intervention Studies Organised by Patient Group

\begin{tabular}{lcccccc}
\hline & $k$ & $N(\mathrm{Nt} / \mathrm{Nc})$ & $\mathrm{SMD}(\mathrm{Cl})$ & $Z$ & $\chi^{2}$ & $N_{\mathrm{fs}}$ \\
\hline Schizophrenia spectrum disorders & & & & & & \\
Targeted, social cognition & 7 & $243(133 / 110)$ & $1.20(0.87-1.54)$ & $7.05^{* * *}$ & 8.20 & 182 \\
Comprehensive, social cognition & 14 & $438(220 / 216)$ & $0.63(0.34-0.92)$ & $4.22^{* * *}$ & $26.83^{*}$ & 177 \\
Broad-based, social cognition & 9 & $571(311 / 260)$ & $0.67(0.43-0.90)$ & $5.52^{* * *}$ & 13.89 & 172 \\
Autism spectrum disorders & & & & & \\
Targeted, social cognition & 3 & $77(37 / 40)$ & $0.41(-0.18-1.00)$ & 1.36 & 2.90 & 2 \\
Comprehensive, social cognition & 1 & $11(6 / 5)$ & $0.53(-0.69-1.74)$ & 0.85 & - & 0 \\
Broad-based, social cognition & 0 & - & - & - & - & - \\
Acquired brain injury & & & & & \\
Targeted, social cognition & 5 & $107(53 / 54)$ & $0.56(0.11-1.01)$ & $2.42^{*}$ & 4.76 & 13 \\
Comprehensive, social cognition & 0 & - & - & - & - & - \\
Broad-based, social cognition & 1 & $26(13 / 13)$ & $0.47(-0.32-1.25)$ & 1.17 & - \\
\hline
\end{tabular}

Note: $k$, number of studies; $N$, number of patients; $\mathrm{Nt}$, number of patients in treatment group; $\mathrm{Nc}$, number of patients in control group; $Z$, significance test; $\chi^{2}$, heterogeneity statistic; Nfs, number of null findings needed for a non-significant combined effect size calculated by the method of Rosenthal (1979).

${ }^{*} p \leq .05,{ }^{* *} p<.01,{ }^{* * *} p<.001$.

SSD, targeted interventions had a large effect on $\mathrm{SC}(\mathrm{SMD}=1.20,95 \% \mathrm{CI}=.87-1.54, Z=7.05$, $p<.001$ ), whilst both comprehensive and broad-based interventions had a moderate effect on $\mathrm{SC} \quad\left(\mathrm{SMD}_{\text {comprehensive }}=.63\right.$, 95\% $\mathrm{CI}=.34-.92, \quad \mathrm{Z}=4.22, p<.001$; $\left(\mathrm{SMD}_{\text {broad-based }}=.67,95 \% \mathrm{CI}=.43-.90, Z=5.52\right.$, $p<.001)$.

Regarding patients with ASD, no significant effects on SC were found for both targeted and comprehensive interventions $\left(\mathrm{SMD}_{\text {targeted }}=.41,95 \% \mathrm{CI}=-.18-1.00, \mathrm{Z}=1.36\right.$, $p=.17 ; \mathrm{SMD}_{\text {comprehensive }}=.53,95 \% \mathrm{CI}=-.69$ $1.74, \mathrm{Z}=.85, p=.40)$. No broad-based interventions for patients with ASD met the criteria for inclusion.

In patients with $\mathrm{ABI}$, a moderate effect of targeted interventions was found on $\mathrm{SC}(\mathrm{SMD}=.56$, $95 \% \mathrm{CI}=.11-1.01, \mathrm{Z}=2.42, p=.02)$. No significant effect on SC was found for broad-based interventions $(\mathrm{SMD}=.47,95 \% \mathrm{CI}=-.32-1.25$, $\mathrm{Z}=1.17, p=.24)$. No comprehensive interventions for patients with $\mathrm{ABI}$ met the inclusion criteria.

\section{Discussion}

To our knowledge, this is the first systematic review that meta-analysed controlled intervention studies in neuropsychiatric patients targeting SC. The overall results showed that the selected SC interventions had a moderate-to-large effect on improving SC in general. The interventions were most effective in improving emotion perception, whilst ToM, social perception, and social functioning could be effectively improved by these treatments as well, with moderate effect sizes. The moderate-to-large effect size for emotion perception is in accordance with the findings from previous systematic reviews in patients with SSD (Fiszdon \& Reddy, 2012; Kurtz \& Richardson, 2011). The effects for ToM and social perception found in the current meta-analyses, being larger than in the aforementioned reviews, could be explained both by the inclusion of more recent studies as well as the inclusion of intervention studies for other neuropsychiatric patient populations. Overall, the meta-analyses showed that targeted interventions were most effective, with a large effect size, in improving SC, followed by broad-based and comprehensive interventions, both with moderate effect sizes. Social perception and ToM were trained most effectively by targeted interventions, emotion perception was trained equally effective by both targeted as well as comprehensive treatments, and social functioning by broad-based interventions.

Targeted interventions were most effective in improving SC and showed a large effect on emotion perception. However, these interventions almost exclusively trained emotion perception, often evaluated by outcome measures typically covering this particular process. The large, positive effects on emotion perception may thus result from 'training to the task' rather than being the result of transfer to untrained tasks. The same phenomenon could be identified in the only targeted intervention study focussing on social perception (Garcia et al., 2003). The large effect for ameliorating 


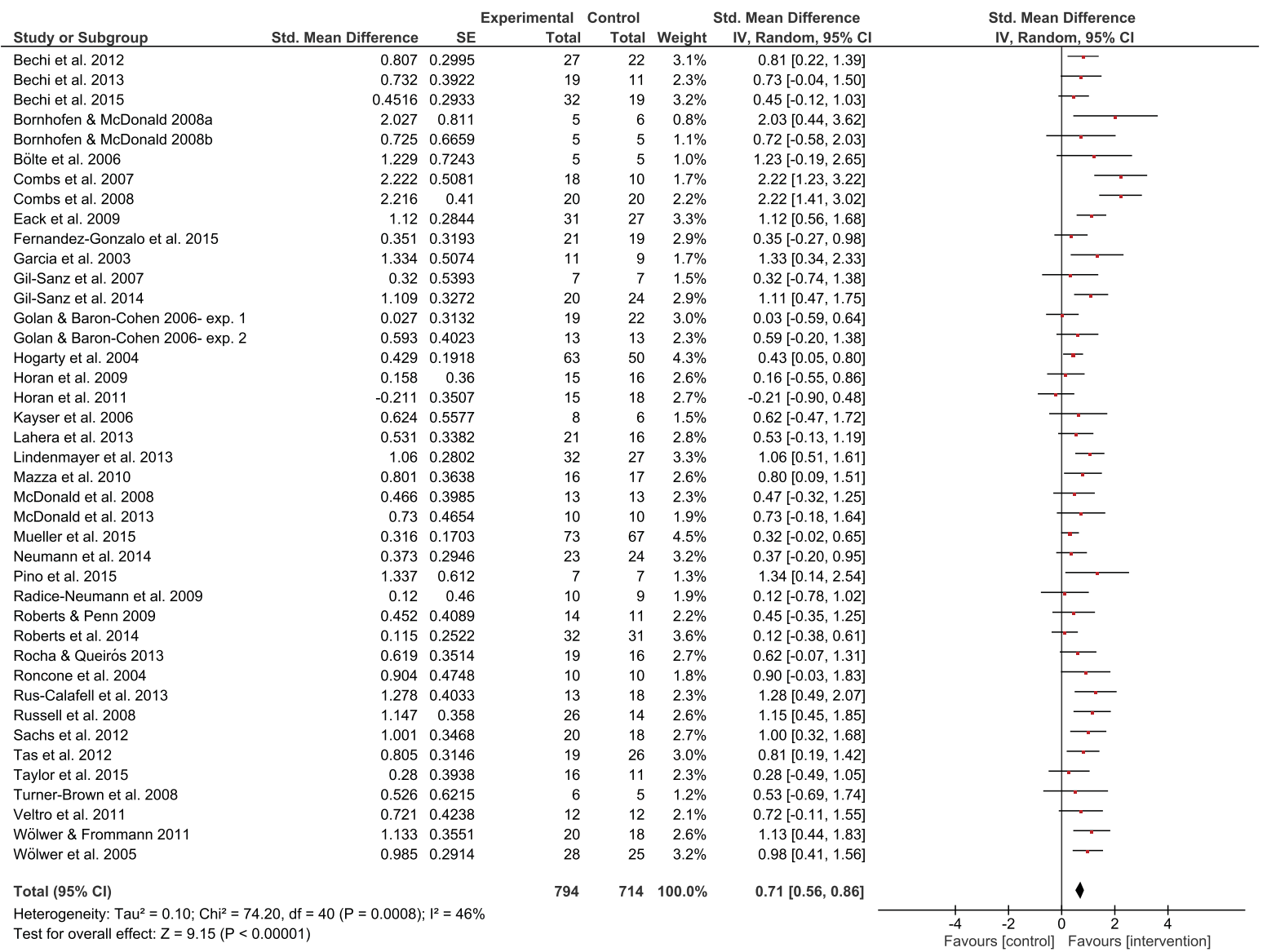

\section{FIGURE 2}

Forest plot of all interventions with social cognition as outcome variable. 


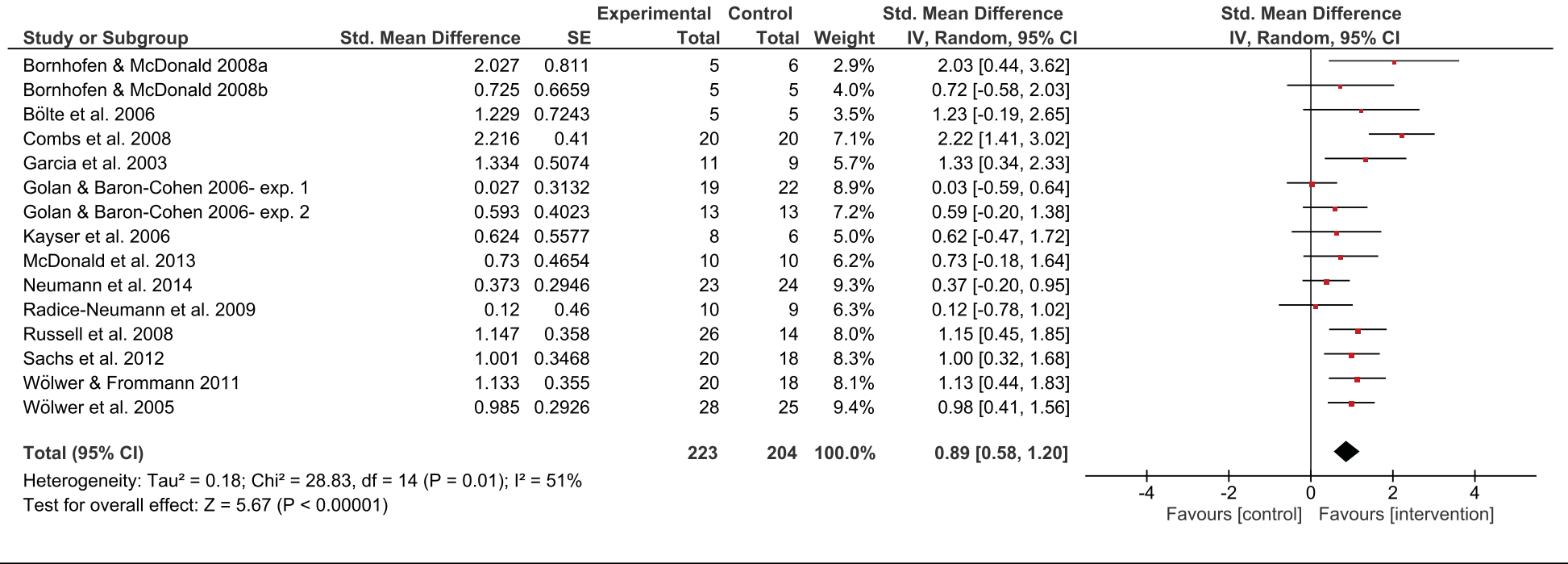

\section{FIGURE 3}

Forest plot of targeted interventions with social cognition as outcome variable. 


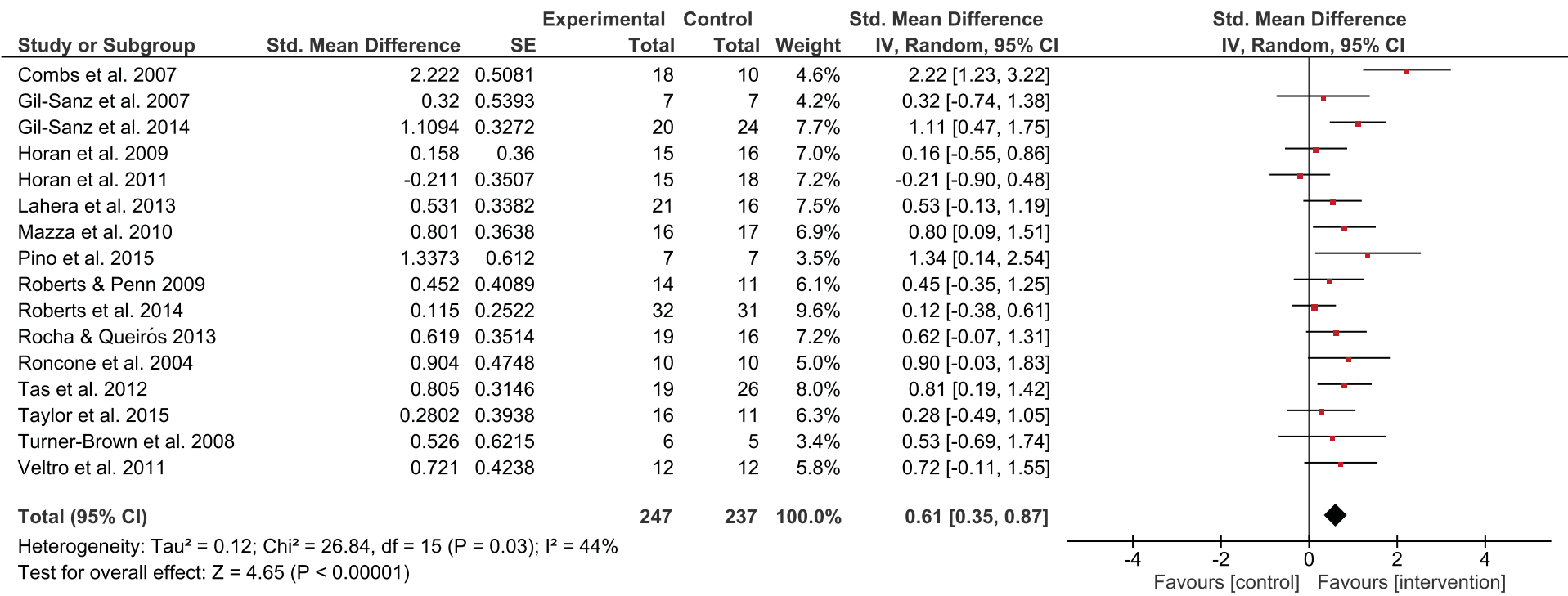

\section{FIGURE 4}

Forest plot of comprehensive interventions with social cognition as outcome variable. 


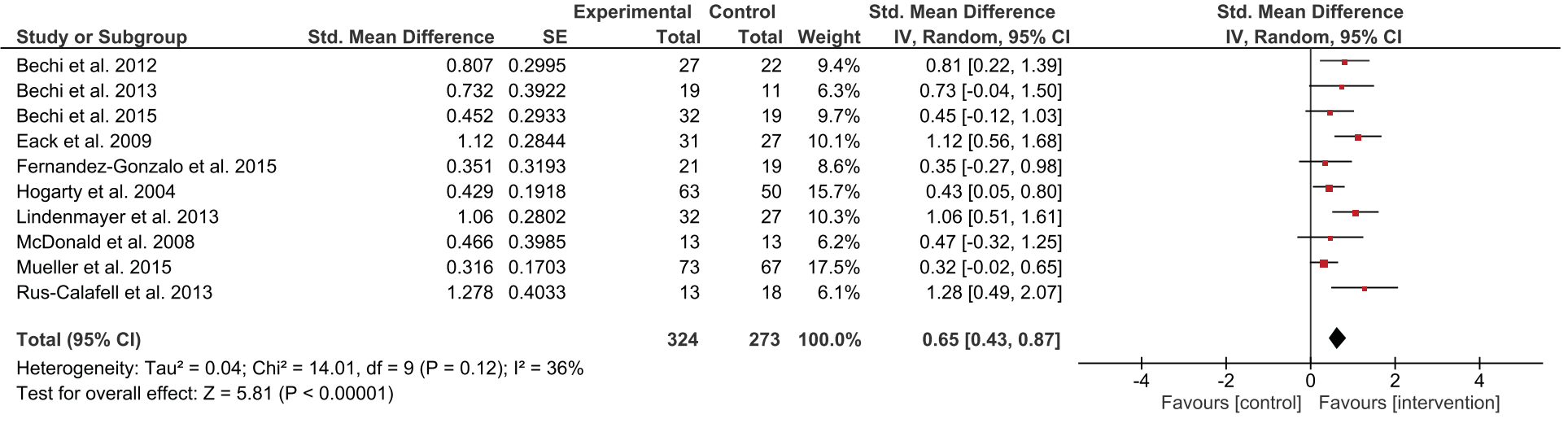

\section{FIGURE 5}

Forest plot of broad-based interventions with social cognition as outcome variable. 
social perception that was found in this study again raises concerns regarding 'training to the task'. Surprisingly, targeted interventions also had a large effect on ToM. Here, only one of the seven targeted interventions measuring ToM was a ToM-intervention (Kayser et al., 2007), the other six focussed on improving emotion perception (Bornhofen \& McDonald, 2008a, 2008b; Golan \& Baron-Cohen, 2006, experiment 1; Golan \& Baron-Cohen, 2006, experiment 2; Neumann et al., 2014; Wölwer \& Fromman, 2011). Whilst this finding suggests that training emotion perception may improve ToM abilities as well, generally, targeted interventions were not able to effectively improve social functioning. However, although only two targeted interventions included a measure of social functioning at post-treatment assessment, a marginally significant, moderate treatment effect was found in the current study.

Comprehensive interventions had a moderate effect on improving SC. The effect size on emotion perception was large, and the effects on ToM and social functioning were moderate. As to the latter, it should be noted that comprehensive interventions, as compared to targeted interventions, are generally performed using longer treatment duration and higher treatment frequency. This relatively higher intensity of the comprehensive interventions may have caused this effect on daily social functioning. In contrast, the comprehensive interventions in the meta-analysis were not able to effectively improve social perception. This is a striking finding, because 6 out of the 16 comprehensive interventions focussed on social perception. After further inspection of these studies, it appeared that only four interventions measured social perception after treatment. Hence, the effects of the social perception modules in comprehensive interventions could be underestimated.

Broad-based interventions had a moderate effect on improving SC in general. These interventions showed a moderate-to-large effect size in improving social functioning. Broad-based interventions were more effective in improving social functioning than targeted and comprehensive interventions. One explanation for this effect may be found in the content of the broad-based interventions. Combining SC treatment with training of neurocognition or social skills may have resulted in more robust effects and a better generalisation to daily life. A second explanation for the transfer effects of broad-based interventions to daily social functioning concerns the intensity of the training. In general, treatment duration of broad-based interventions is considerably longer than that of targeted and comprehensive interventions, and broad-based interventions include more sessions. Roberts et al. (2014) suggested that more frequent sessions may be more effective for maximising treatment benefits. It could be hypothesised that patients participating in more intensive intervention programmes have had more opportunities to practise and incorporate new knowledge and skills in their daily life. The importance of practise opportunities was also discussed by Fiszdon and Reddy (2012). They suggested that higherorder SC functions might be more impacted by intensive treatment. Apart from their impact on social functioning, broad-based interventions were moderately effective in improving ToM and emotion perception. Notably, of the broad-based intervention studies that included social perception as a target of treatment, only one actually measured social perception. Here, the effect was small and not statistically significant.

Most studies have been performed in patients with SSD. The small number of studies on adult patients with ASD was somewhat surprising. That is, during the literature search, many SC intervention studies for patients with ASD were identified, but almost all of the interventions focussed on children and were therefore not eligible (see e.g. Fletcher-Watson et al., 2014). Furthermore, only in the group of patients with SSD, all three intervention types were studied. For patients with ASD and ABI, mainly targeted interventions have been employed. In these latter patient groups, more research is needed on the effects of (other) SC interventions. Targeted interventions appeared to be most effective for training SC in patients with SSD, with large effect sizes. This finding is in accordance with the results from previous reviews (Choi et al., 2009; Horan et al., 2008; Kurtz et al., 2011; Mueller et al., 2013; Statucka \& Walder, 2013). Broadbased interventions were slightly more effective than comprehensive interventions, both with moderate effect sizes.

In patients with ASD, small to moderate effects on SC were found for targeted and comprehensive interventions, but these effects were not statistically significant. No broad-based interventions for SC in ASD could be included in the meta-analyses. The non-significant results could reflect a power problem in the original studies. This idea is strengthened by the fact that the present lack of significant treatment effects contrasts with the results of the systematic review of BishopFitzpatrick et al. (2013), in which psychosocial and SC interventions were shown to be effective in an adult ASD population. Their systematic review included more studies ( $k=13$ with $N=291$ patients), hence had more statistical power. However, also non-controlled studies and case-studies were included, which could in turn have led to an over- 
estimation of the actual effects. The meta-analysis of Fletcher-Watson et al. (2014) also showed larger effect sizes in a mixed population of children and adults with ASD.

Regarding patients with $\mathrm{ABI}$, only targeted interventions had a moderate, significant effect on SC. In one study that investigated the effects of a broad-based intervention, effects were smallmoderate, but statistically insignificant. Previous review articles regarding patients with $\mathrm{ABI}$ also found positive indications for the effectiveness of SC interventions on SC (Braden, 2014; Driscoll et al., 2011; Manly \& Murphy, 2012).

Only 10 of the 41 studies in the meta-analyses included a follow-up assessment after treatment had ended. Although the interval durations were highly variable, it appeared that most interventions were able to maintain at least one of the effects at follow-up. The majority of the followup assessments were performed as an evaluation of a targeted intervention, suggesting that focussed and relatively brief interventions are not only effective in improving the performance on several SC tasks directly post-treatment, but that this improvement remains after a period without treatment.

Interestingly, no controlled intervention studies have focussed on the reduction of alexthymia in adult neuropsychiatric patients yet. Most alexithymia intervention studies published so far focussed on healthy participants or individuals with somatic disorders. Moreover, alexithymia was frequently studied as a moderator for treatment success, rather than a primary outcome variable. Since alexithymia has been related to lower levels of quality of life, more problems in social relations, somatic complaints, and psychiatric disorders (Kennedy \& Franklin, 2002; Taylor \& Bagby, 2012), it seems to be a very relevant treatment target for future studies.

The results of this meta-analysis have implications for future research. More studies should focus on SC interventions in other neuropsychiatric disorders than SSD, such as in adults with ASD and ABI. Patients with other neuropsychiatric disorders that comprise SC problems may also benefit from SC interventions, such as those with mood or anxiety disorders, neurodegenerative conditions, as well as patients with SC deficits caused by a genetic syndrome. Principally, the large treatment effects of the available interventions show that these are appropriate treatments for enhancing SC, that may also be suitable for adjustment and implementation in new patient groups. Future interventions should also pay attention to alexithymia, given the high correlations with other psychological and somatic problems. Lastly, it is advised to also in- clude measures of social perception to evaluate the effects of an SC intervention.

The results of this study should be interpreted in light of its strengths and weaknesses. Strengths of this meta-analysis include the large sample size of controlled intervention studies $(k=41$ studies with a total of $N=1,508$ patients). Also, the failsafe $N$ for the main meta-analyses showed that a large number of unpublished studies with null findings should exist to result in insignificant effect sizes, which is unlikely. Furthermore, this is the first study that meta-analysed the effects of three different SC treatment types on a population of neuropsychiatric patients instead of one specific patient group. At the same time, this explains the high degree of heterogeneity. A recurrent issue in many meta-analyses is the notable amount of differences found in patient population, intervention type, control group, and intervention content. In the present study, we partly succeeded in reducing this heterogeneity by performing different meta-analyses for the various treatment types and patient populations. Although the three categories of interventions (targeted, comprehensive, and broad-based) are well defined, there is some overlap between the three subtypes and other categorisations can be made. As to the patient groups, only three could be included in the meta-analysis. Although other psychiatric and neurological conditions met the definition of a neuropsychiatric disorder (e.g., mood and anxiety disorders, dementia, or Huntington's disease), the literature search did not return eligible SC intervention studies for these populations, despite the fact that SC disorders have been described in these conditions (Bediou et al., 2009; Gregory et al., 2002; Lough et al., 2006; Plana et al., 2014; Samamé, 2013; Snowden et al., 2003). Patients with SSD were overrepresented in this meta-analysis. This may have implications for the generalisation to the overall population of patients with neuropsychiatric disorders. Furthermore, it is conceivable that there are differences in the specific SC problems between the three patient groups, which could have influenced treatment effectiveness.

In conclusion, the current meta-analysis showed that SC interventions are effective in improving SC in neuropsychiatric patients. Targeted interventions are effective in improving task performance of the trained SC domains (mainly emotion perception), but they are also able to positively affect ToM. Follow-up assessments in a small group of mainly targeted interventions suggested that some treatment effects remained after the intervention had ended. Comprehensive interventions were able to improve emotion perception, ToM, and social functioning. The broad-based 
interventions were especially effective in improving social functioning, probably due to more extensive treatment content and a more intensive design. Although our meta-analytic approach could not isolate effective elements specifically, training of emotion perception appears an important ingredient of effective SC interventions. Treatments should also incorporate social functioning and provide sufficient practise opportunities in daily life in order to optimise generalisation and maintenance of effects.

\section{Financial Support}

This research received no specific grant from any funding agency, commercial or not-for-profit sectors.

\section{Conflicts of Interest}

None.

\section{Ethical Standards}

Not applicable.

\section{References}

Adolphs, R. (2001). The neurobiology of social cognition. Current Opinion in Neurobiology, 11(2), 231239.

Ambady, N., Hallahan, M., \& Rosenthal, R. (1995). On judging and being judged accurately in zero acquaintance situations. Journal of Personality and Social Psychology, 69(3), 519-529.

Bähler, M. (2012). Evaluation eines neu Entwickelten Fragebogen zur Emotionserkennung bei Schizophren Erkrankten. Unpublished master thesis, Université de Fribourg, Switserland.

Bangalore, N.G., \& Varambally, S. (2012). Yoga therapy for schizophrenia. International Journal of Yoga, 5(2), 85-91.

Banse, R., \& Scherer, K.R. (1996). Acoustic profiles in vocal emotion expression. Journal of Personality and Social Psychology, 70(3), 614-636.

Baron-Cohen, S., \& Wheelwright, S. (2004). The empathy quotient: An investigation of adults with Asperger syndrome or high functioning autism, and normal sex differences. Journal of Autism and Developmental Disorders, 34(2), 163-175.

Baron-Cohen, S., Wheelwright, S., Hill, J.J., Raste, Y., \& Plumb, I. (2001). The "reading the mind in the eyes" test - revised version: A study with normal adults, and adults with Asperger syndrome or highfunctioning autism. Journal of Child Psychology and Psychiatry, 42(2), 241-251.

Baurain, C., \& Nader-Grosbois, N. (2013). Theory of mind, socio-emotional problem-solving, socio- emotional regulation in children with intellectual disability and in typically developing children. Journal of Autism and Developmental Disorders, 43(5), 1080-1097.

Beauchamp, H.M., \& Anderson, V. (2010). SOCIAL: An integrative framework for the development of social skills. Psychological Bulletin, 136(1), 39-64.

Bechi, M., Bosia, M., Spangaro, M., Buonocore, M., Cocchi, F., Pigoni, A., ... Cavallaro, R. (2015). Combined social cognitive and neurocognitive rehabilitation strategies in schizophrenia: Neuropsychological and psychopathological influences on Theory of Mind improvement. Psychological Medicine, 45(2), 3147-3157.

Bechi, M., Riccaboni, R., Ali, S., Fresi, F., Buonocore, M., Bosia, M., ... Cavallaro, R. (2012). Theory of mind and emotion processing training for patients with schizophrenia: Preliminary findings. Psychiatry Research, 198(3), 371-377.

Bechi, M., Spangaro, M., Bosia, M., Zanoletti, A., Fresi, F., Buonocore, M., .. . Cavallaro, R. (2013). Theory of Mind intervention for outpatients with schizophrenia. Neuropsychological Rehabilitation, 23(3), 383400.

Bediou, B., Ryff, I., Mercier, B., Milliery, M., Hénaff, M., D'Amato, T., ... Krolak-Salmon, P. (2009). Impaired social cognition in mild Alzheimer disease. Journal of Geriatric Psychiatry and Neurology, 22, 130-140.

Beer, J.S., \& Ochsner, K.N. (2006). Social cognition: A multi-level analysis. Brain Research, 1079(1), 98105.

Bell, M., Bryson, G., \& Lysaker, P. (1997). Positive and negative affect recognition in schizophrenia: A comparison with substance abuse and normal control subjects. Psychiatry Research, 73(1), 73-82.

Birchwood, M., Smith, J., Cochrane, R., Wetton, S., \& Copestake, S. (1990). The social functioning scale: The development and validation of a new scale of social adjustment for use in family intervention programmes with schizophrenic patients. British Journal of Psychiatry, 157(6), 853-859.

Bird, G., \& Cook, R. (2013). Mixed emotions: The contribution of alexithymia to the emotional symptoms of autism. Translational Psychiatry, 3(7), 1-8.

Bishop-Fitzpatrick, L., Minshew, N.J., \& Eack, S.M. (2013). A systematic review of psychosocial interventions for adults with autism spectrum disorders. Journal of Autism and Developmental Disorders, 43(3), 687-694.

Blair, R.J., \& Cipolotti, L. (2000). Impaired social response reversal: A case of 'acquired sociopathy'. Brain, 123(6), 135-147.

Bölte, S., Feineis-Matthews, S., Leber, S., Dierks, T., Hubl, D., \& Poustka, F. (2002). The development and evaluation of a computer-based program to test and to teach the recognition of facial affect. International Journal of Circumpolar Health, 61(suppl. 2), 61-68. 
Bölte, S., Feineis-Matthews, S., Prvulovic, D., Hubl, D., Dierks, T., \& Poustka, F. (2006). Facial affect recognition training in autism: Can we animate the fusiform gyrus? Behavioral Neuroscience, 120(1), 211-216.

Bora, E., Eryavuz, A., Kayahan, B., Sungu, G., \& Veznedaroglu, B. (2008). Empathic abilities in people with schizophrenia. Psychiatry Research, 160(1), 23-29.

Bornhofen, C., \& McDonald, S. (2008a). Treating deficits in emotion perception following traumatic brain injury. Neuropsychological Rehabilitation, 18(1), 2244.

Bornhofen, C., \& McDonald, S. (2008b). Comparing strategies for treating emotion perception deficits in traumatic brain injury. Journal of Head Trauma Rehabilitation, 23(2), 103-115.

Braden, C. (2014). Communication and social skills training. In S. McDonald, L. Togher, \& C. Code (Eds.), Social and communication disorders following traumatic brain injury (2nd ed., pp. 307-335). East Sussex: Psychology Press.

Brüne, M. (2003a). Social cognition and behavior in schizophrenia. In W. Schiefenhövel (Ed.), The social brain-evolution and pathology (pp. 277-313). Chichester, UK: John Wiley \& Sons.

Brüne, M. (2003b). Theory of Mind and role of IQ in chronic disorganized schizophrenia. Schizophrenia Research, 60(1), 57-64.

Brüne, M. (2005). Emotion recognition, 'theory of mind', and social behavior in schizophrenia. Psychiatry Research, 133(2), 135-147.

Burnett, A.C., Reutens, D.C., \& Wood, A.G. (2010). Social cognition in Turner's syndrome. Journal of Clinical Neuroscience, 17(3), 283-286.

Byrne, G., Bogue, J., Egan, R., \& Lonergan, E. (2014). "Identifying and describing emotions": Measuring the effectiveness of a brief alexithymia-specific, intervention for sex offender population. Sexual Abuse: A Journal of Research and Treatment, 28, 599-619. doi: 10.1177/1079063214558940.

Cameron, K., Ogrodniczuk, J., \& Hadjipavlou, G. (2014). Changes in alexithymia following psychological intervention: A review. Harvard Reviews of Psychiatry, 22(3), 162-178.

Choi, J.H., Kim, J.H., Lee, J., \& Green, M.F. (2009). Social cognition training for individuals with schizophrenia: A review of targeted interventions. Clinical Psychopharmacology and Neuroscience, 7(2), 29-38.

Combs, D.R., Adams, S.D., Penn, D.L., Roberts, D., Tiegreen, J., \& Stem, P. (2007). Social Cognition and Interaction Training (SCIT) for inpatients with schizophrenia spectrum disorders: Preliminary findings. Schizophrenia Research, 91(1), 112-116.

Combs, D.R., Tosheva, A., Penn, D.L., Basso, M.R., Wanner, J.L., \& Laib, K. (2008). Attentional shaping as a means to improve emotion perception deficits in schizophrenia. Schizophrenia Research, 105(1), 68-77.
Corbett, B.A., Gunther, J.R., Comins, D., Price, J., Ryan, N., Simon, D., ... Rios, T. (2011). Brief report: Theatre as therapy for children with autism spectrum disorders. Journal of Autism and Developmental Disorders, 41(4), 505-511.

Corcoran, R., Mercer, G., \& Firth, C. (1995). Schizophrenia, symptomatology and social inference: Investigating "theory of mind" in people with schizophrenia. Schizophrenia Research, 17(1), 5-13.

Cornblatt, B.A., Auther, A.M., Niendam, T., Smith, C.W., Zinberg, J., Bearden, C.E., \& Cannon, T.D. (2007). Preliminary findings for two new measures of social and role functioning in the prodromal phase of schizophrenia. Schizophrenia Bulletin, 33(3), 688-702.

Couture, S.M., Penn, D.L., Losh, M., Adolphs, R., Hurley, R., \& Piven, J. (2010). Comparison of social cognitive functioning in schizophrenia and high functioning autism: More convergence than divergence. Psychological Medicine, 40(4), 569579.

Couture, S.M., Penn, D.L., \& Roberts, D.L. (2006). The functional significance of social cognition in schizophrenia: A review. Schizophrenia Bulletin, 32(suppl. 1), 44-63.

Croker, V. \& McDonald, S. (2005). Recognition of emotion from facial expression following traumatic brain injury. Brain Injury, 19(10), 787-799.

Curran, J.P. (1982). A procedure for the assessment of social skills: The simulated social interactions test. In J.P. Curran, \& P.M. Monti (Eds.), Social skills training: A practical handbook for assessment and treatment (pp. 313-347). New York: Guilford Press.

Davis, M.H. (1983). Measuring individual differences in empathy: Evidence for a multidimensional approach. Journal of Personality and Social Psychology, 44(1), 113-125.

Deeks, J.J., Higgins, J. P.T., \& Altman, D.G. (2008). Analysing data and undertaking meta-analyses. In J.P.T. Higgins \& S. Green (Eds.), Cochrane handbook for systematic reviews of interventions (version 5.1.0, pp. 243-297). The Cochrane Collaboration, Retrieved from www.cochrane-handbook.org. West Sussex: John Wiley \& Sons Ltd.

Dimoska, A., McDonald, S., Pell, M.C., Tate, R.L., \& James, C.M. (2010). Recognising vocal expressions of emotion following traumatic brain injury: Is this 'what' more important than the 'how'? Journal of the International Neuropsychological Society, 16(2), 369-382.

Driscoll, D.M., Dal Monte, O., \& Grafman, J. (2011). A need for improved training interventions for the remediation of impairments in social functioning following brain injury. Journal of Neurotrauma, 28(2), 319-326.

Dyck, M.J., Ferguson, K., \& Shochet, I.M. (2001). Do autism spectrum disorders differ from each other and from non-spectrum disorders on emotion recognition tests? European Child \& Adolescent Psychiatry, 10(2), 105-116. 
Dywan, J., Roden, R., \& Murphy, T. (1995). Orbitofrontal symptoms are predicted by mild head injury among normal adolescents. Journal of the International Neuropsychological Society, 1(121), b10.

Eack, S.M., Greenwald, D.P., Hogarty, S.S., Cooley, S.J., DiBarry, A.L., Montrose, D.M., \& Keshavan, M.S. (2009). Cognitive enhancement therapy for earlycourse schizophrenia: Effects of a two-year randomized controlled trial. Psychiatric Services, 60(11), 1468-1476.

Egger, J.I., Zwanenburg, R.J., Van Ravenswaaij-Arts, C.M., Kleefstra, T., \& Verhoeven, W.M. (2016). Neuropscyhological phenotype and psychoathology in seven adult patients with Phelan-McDermid syndrome: Implications for treatment strategy. Genes, Brain and Behavior, 15(4), 395-404.

Ekman, P., \& Friesen, W. (1976). Pictures of facial affect. Palo Alto, CA: Consulting Psychologists Press.

Farrell, A.D., Rabinowitz, J.A., Wallander, J.L., \& Curran, J.P. (1985). An evaluation of two formats for the intermediary level assessment of social skills. Journal of Psychopathology and Behavioral Assessment, 7(2), 155-171.

Fernandez-Gonzalo, S., Turan, M., Jodar, M., Pousa, E., Hernandez Rambla, C., Garcia, R., \& Palao, D. (2015). A new computerized cognitive and social cognition training specifically designed for patients with schizophrenia/schizo-affective disorder in early stages of illness: A pilot study. Psychiatry Research, 228(3), 501-509.

Fett, A.J., Viechtbauer, W., Dominguez, M., Penn, D.L., Van Os, J., \& Krabbendam, L. (2011). The relationship between neurocognition and social cognition with functional outcomes in schizophrenia: A metaanalysis. Neuroscience and Biobehavioral Reviews, 35(3), 573-588.

Fiske, S.T., \& Taylor, S.E. (2013). Social cognition, from brains to culture (2nd ed.). London: SAGE Publications Ltd.

Fiszdon, J.M., \& Reddy, L.F. (2012). Review of social cognitive treatments for psychosis. Clinical Psychology Review, 32(8), 724-740.

Fletcher-Watson, S., McConell, F., Manola, E., \& McConachie, H. (2014). Interventions based on the Theory of Mind cognitive model for autism spectrum disorders (ASD). Cochrane Database of Systematic Reviews, 3, 1-82. doi: 10.1002/14651858.CD008785.pub2.

Frith, C.D., \& Corcoran, R. (1996). Exploring 'theory of mind' in people with schizophrenia. Psychological Medicine, 26(3), 521-530.

Fuentes, I., Garcia, S., Ruiz, J.C., Soler, M.J., \& Roder, V. (2007). Social perception training in schizophrenia: A pilot study. International Journal of Psychology and Psychological Therapy, 7(1), 1-12.

Gambrill, E.D., \& Richey, C.A. (1975). An assertion inventory for use in assessment and research. Behavior Therapy, 6(4), 550-561.
Garcia, S., Fuentes, I., Ruiz, J.C., Gallach, E., \& Roder, V. (2003). Application of the IPT in a Spanish sample: Evaluation of the "social perception subprogramme". International Journal of Psychology and Psychological Therapy, 3(2), 299-310.

Gil-Sanz, D., Diego-Lorenzo, M., Bengochea-Seco, R., Arrieta-Rodríguez, M., Lastra-Martínez, I., Sánchez-Calleja, R., \& Álvarez-Soltero, A. (2009). Efficacy of a social cognition training program for schizophrenic patients: A pilot study. The Spanish Journal of Psychology, 12(1), 184-191.

Gil-Sanz, D., Fernández-Modamio, M., BengocheaSeco, R., Arrieta-Rodríguez, M., \& Pérez-Fuentes, G. (2014). Efficacy of the social cognition training program in a sample of outpatients with schizophrenia. Clinical Schizophrenia \& Related Psychoses, 1-27, doi: 10.3371/CSRP.GIFE.013114.

Golan, O., \& Baron-Cohen, (2006). Systemizing empathy: Teaching adults with Asperger syndrome or high-functioning autism to recognize complex emotions using interactive media. Development and Psychopathology, 18(2), 591-617.

Golan, O., Baron-Cohen, S., \& Hill, J.J. (2006). The cambridge mindreading (CAM) face-voice battery: Testing complex emotion recognition in adults with and without Asperger Syndrome. Journal of Autism and Developmental Disorders, 36(2), 169-183.

Golan, O., Baron-Cohen, S., Hill, J.J., \& Golan, Y. (2006). The 'reading the mind in films' task: Complex emotion recognition in adults with and without autism spectrum conditions. Social Neuroscience, 1(2), 111-123.

Golan, O., Baron-Cohen, S., Hill, J.J., \& Rutherford, M.D. (2006). The "reading the mind in the voice test-revised": A study of complex emotion recognition in adults with and without autism spectrum conditions. Journal of Autism and Developmental Disorders, 37(6), 1096-1106.

Green, M.F., Olivier, B., Crawley, J.N., Penn, D.L., \& Silverstein, S. (2005). Social cognition In schizophrenia: Recommendations from the measurement and treatment research to improve cognition in schizophrenia new approaches conference. Schizophrenia Bulletin, 31(4), 882-887.

Green, R.E.A., Turner, G.R., \& Thompson, W.F. (2004). Deficits in facial emotion perception in adults with recent traumatic brain injury. Neuropsychologia, 42(2), 133-141.

Gregory, C., Lough, S., Stone, V., Erzinclioglu, S., Martin, L., Baron-Cohen, S., \& Hodges, J.R. (2002). Theory of mind in patients with frontal variant frontotemporal dementia and Alzheimer's disease theoretical and practical implications. Brain, 125(4), $752-764$.

Grynberg, D., Chang, B., Corneille, O., Maurage, P., Vermeulen, N., Berthoz, S., \& Luminet, O. (2012). Alexithymia and the processing of emotional facial expressions (EFEs): Systematic review, unanswered questions and further perspectives. PLOS ONE, 7(8), $1-20$. 
Guastella, A.J., \& MacLeod, C. (2012). A critical review of the influence of oxytocin nasal spray on social cognition in humans: Evidence and future directions. Hormones and Behavior, 61(3), 410 418.

Happe, F.G. (1994). An advanced test of theory of mind: Understanding of story characters' thoughts and feelings by able autistic, mentally handicapped, and normal children and adults. Journal of Autism and Developmental Disorders, 24(2), 129-154.

Healey, K., Roberts, D., Combs, D., \& Penn, D. (2012, November). Observable Social Cognition: A Rating Scale: An Interview-Based Assessment for Schizophrenia. Paper presented at the annual meeting of the Association for Behavioral and Cognitive Therapies, National Harbor, MD.

Hogarty, G.E., \& Flesher, S. (1999). Practice principles of cognitive enhancement therapy for schizophrenia. Schizophrenia Bulletin, 25(4), 693-708.

Hogarty, G.E., Flesher, S., Ulrich, R., Carter, M., Greenwald, D., Pogue-Geile, M., ... Zorretich, R. (2004). Cognitive enhancement therapy for schizophrenia: Effects of a 2-year randomized trial on cognition and behavior. Archives of General Psychiatry, 61(9), 866-876.

Hogarty, G.E., \& Greenwald, D.P. (2006). Cognitive Enhancement Therapy: The training manual. Pittsburgh, PA: University of Pittsburgh Medical Center, Retrieved from http://www. CognitiveEnhancementTherapy.com.

Hogarty, G.E, Greenwald, D.P., \& Eack, S.M. (2006). Durability and mechanism of effect of cognitive enhancement therapy. Psychiatric Services, 57(12), 1751-1757.

Horan, W.P., Kern, R.S., \& Green, M.F. (2008). Social cognition training for individuals with schizophrenia: Emerging evidence. American Journal of Psychiatric Rehabilitation, 11(3), 205-252.

Horan, W.P., Kern, R.S., Shokat-Fadai, K., Sergi, M.J., Wynn, J.K., \& Green, M.F. (2009). Social cognitive skills training in schizophrenia: An initial efficacy study of stabilizes outpatients. Schizophrenia Research, 107(1), 47-54.

Horan, W.P., Kern, R.S., Tripp, C., Hellemann, G., Wynn, J.K., Bell, M., ... Green, M.F. (2011). Efficacy and specificity of Social Cognitive Skills Training for outpatients with psychotic disorders. Journal of Psychiatric Research, 45(8), 1113-1122.

Hornak, J., Rolls, E.T., \& Wade, D. (1996). Face and voice expression identification in patients with emotional and behavioural changes following ventral lobe damage. Neuropsychologia, 34(4), 247-261.

Höschel, K., \& Irle, E. (2001). Emotional priming of facial affect identification in schizophrenia. Schizophrenia Bulletin, 27(2), 317-327.

Hsieh, P.C., Huang, H.Y., Wang, H.C., Liu, Y.C., Bai, Y.M., Chen, K.C., \& Yang, Y.K. (2011). Intercorrelations between the personal and social performance scale, cognitive function, and activities of daily liv- ing. Journal of Nervous and Mental Disease, 154(7), $1-63$.

Kayser, N., Sarfati, Y., Besche, C., \& Hardy-Baylé, M. (2007). Elaboration of a rehabilitation method based on a pathogenetic hypothes of "theory of mind" impairment in schizophrenia. Neuropsychological Rehabilitation, 16(1), 83-95.

Kennedy, M., \& Franklin, J. (2002). Skill-based treatment for alexithymia: An exploratory case series. Behaviour Change, 19(3), 158-171.

Kerr, S.L., \& Neale, J.M. (1993). Emotion perception in schziphrenia: Specific deficit or further evidence of generalized poor performance? Journal of Abnormal Psychology, 102(2), 312-318.

Kohler, C.G., Anselmo-Gallagher, G., Bikler, W., Karlawish, J., Gur, R.E., \& Clark, C.M. (2005). Emotion-discrimination deficits in mild Alzheimer disease. American Journal of Geriatric Psychiatry, 13(11), 926-933.

Kurtz, M., \& Richardson, C.I. (2011). Social cognitive training for schizophrenia: A meta-analytic investigation of controlled research. Schizophrenia Bulletin, 38(5), 1092-1104.

Lahera, G., Benito, A., Montes, J.M., Fernández-Liria, Olbert, C.M., \& Penn, D.L. (2013). Journal of Affective Disorders, 146(1), 132-136.

Lane, R.D., Quinlan, D.M., Schwartz, G.E., Walker, P.A., \& Zeitlin, S.B. (1990). The levels of emotional awareness scale: A cognitive-developmental measure of emotion. Journal of Personality Assessment, 55(1-2), 124-134.

Lane, R.D., Sechrest, L., Riedel, R., Shapiro, D.E., \& Kasniak, A.W. (2000). Pervasive emotion recognition deficit common to alexithymia and the repressive coping style. Psychosomatic Medicine, 62(3), 492-501.

Lane, R.D., Sechrest, L., Reidel, R., Weldon, V., Kaszniak, A., \& Schwartz, G.E. (1996). Impaired verbal and nonverbal emotion recogniton in alexithymia. Psychosomatic Medicine, 58(4), 203-210.

Leffert, J.S., Siperstein, G.N. \& Widaman, K.F. (2010). Social perception in children with intellectual disabilities: The interpretation of benign and hostile intentions. Journal of Intellectual Disability Research, 54(2), 168-180.

Lerner, M.D., Mikami, A.Y., \& Levine, K. (2010). Sociodramatic affective-relational intervention for adolescents with Asperger syndrome \& high functioning autism: Pilot study. Autism, 15, 21-42. doi: 10.1177/1362361309353613.

Lindenmayer, J., McGurk, S.R., Khan, A., Kaushik, S., Thanju, A., Hoffman, L., ... Herrmann, E. (2013). Improving social cognition in schizophrenia: A pilot intervention combining computerized social cognition training with cognitive remediation. Schizophrenia Bulletin, 39(3), 507-517.

Lough, S., Kipps, C.M., Treise, C., Watson, P., Blair, J. R., \& Hodges, J. R. (2006). Social reasoning, emotion and empathy in frontotemporal dementia. $\mathrm{Neu}$ ropsychologia, 44(6), 950-958. 
Lowe, M.R., \& Cautela, J.R. (1978). A self-report measure of social skill. Behavior Therapy, 9(4), 535-544.

Maher, C.G., Sherrington, C., Herbert, R.D., Moseley, A.M., \& Elkins, M. (2003). Reliability of the PEDro scale for rating quality of randomized controlled trials. Physical Therapy, 83(8), 713-721.

Manly, T., \& Murphy, F.C. (2012). Rehabilitation of executive function and social cognition impairments after brain injury. Current Opinion in Neurology, 25, 656-661.

Mann, L.S., Wise, T.N., Trinidad, A., \& Kohanski, R. (1995). Alexithymia, affect recognition, and five factors of personality in substance abusers. Perceptual and Motor Skills, 81(1), 35-40.

Martin, J.R., Casas-Anguera, E., Ochoa, S., Escandell, M.J., Prat, G., \& Garcia-Franco, M. (2012, February). Validation of the Spanish version inventory of assertiveness Gambrill and Richeyin populations with schizophrenia. Paper presented at WPA Thematic Conference: Mental Health and Family Medicine Working Together, Granada.

Matsumoto, D., \& Ekman, P. (1988). Japanese and Caucasian Facial Expressions of Emotion (JACFEE). San Francisco, CA: Department of Psychology, San Francisco State University.

Mayer, J.D., Salovey, P., \& Caruso, D. R. (2002). Mayersalovey-caruso emotional intelligence test (MSCEIT) user's manual. Toronto: MHS Publishers.

Mayer, J.D., Salovey, P., Caruso, D.R., \& Sitarenios, G. (2003). Measuring emotional intelligence with the MSCEIT V2.0. Emotion, 3(1), 97-105.

Mazza, M., Costagliola, C., Di Michele, V., Magliani, V., Pollice, R., Ricci, A., ... Galzio, R. J. (2007). Deficit of social cognition in subjects with surgically treated frontal lobe lesions and in subjects affected by schizophrenia. European Archives of Psychiatry and Clinical Neuroscience, 257(1), 12-22.

Mazza, M., Lucci, G., Pacitti, F., Pino, M. C., M., Mariano, M., Cassacchia, \& Roncone, R. (2010). Could schizophrenic subjects improve their social cognition abilities only with observation and imitation of social situations? Neuropsychological Rehabilitation, 20(5), 675-703.

McDonald, S., Bornhofen, C., Shum, D., Long, E., Saunders, C., \& Neulinger, K. (2006). Reliability and validity of 'The Awareness of Social Inference Test (TASIT): A clinical test of social perception. Disability and Rehabilitation, 28(24), 1529-1542.

McDonald, S., Flanagan, S., Rollins, J., \& Kinch, J. (2003). TASIT: A new clinical tool for assessing social perception after traumatic brain injury. Journal of Head Trauma Rehabilitation, 18(3), 219-238.

McDonald, S., Tate, R., Togher, L., Bornhofen, C., Long, E., Gertler, P., \& Bowen, R. (2008). Social skills treatment for people with severe, chronic acquired brain injuries: A multicenter trial. Archives of Physical Medicine and Rehabilitation, 89(9), 1648-1659.
McDonald, S., Togher, L., Tate, R., Randall, R., English, T., \& Gowland, A. (2013). A randomised controlled trial evaluating a brief intervention for deficits in recognising emotional prosody following severe ABI. Neuropsychological Rehabilitation, 23(2), 267-286.

McGann, W., Werven, G., \& Douglas, M.M. (1997). Social competence and head injury: A practical approach. Brain Injury, 11(9), 621-628.

Meyer-Lindenberg, A., Hariri, A.R., Munoz, K.E., Mervis, C.B., Mattay, V.S., Morris, C.A., \& Berman, K.F. (2005). Neural correlates genetically abnormal social cognition in Williams syndrome. Nature Neuroscience, 8(8), 991-993.

Moher, D., Liberati, A., Tetzlaff, J., \& Altman, D.G., The PRISMA Group (2009). Preferred Reporting Items for Systematic Reviews and Meta-Analyses: The PRISMA Statement. PLoS Med, 6(7), 1-6.

Moriguchi, Y., Ohnishi, T., Lane, R.D., Maeda, M., Mori, T., Nemoto, K., ... Komaki, G. (2006). Impaired self-awareness and theory of mind: An fMRI study of mentalizing in alexithymia. NeuroImage, 32(3), 1472-1482.

Morosini, P.L., Magliano, L., Brambilla, L., Ugolini, S., \& Pioli, R. (2000). Development, reliability and acceptability of a new version of the DSM-IV Social and Occupational Functioning Assessment Scale (SOFAS) to assess routine social functioning. Acta Psychiatrica Scandinavica 101(4), 323-329.

Mueller, D.R., Schmidt, S.J., \& Roder, V. (2013). Integrated Psychological Therapy: Effectiveness in schizophrenia inpatient settings related to patients' age. American Journal of Geriatric Psychiatry, 21(3), 231-241.

Mueller, D.R., Schmidt, S.J., \& Roder, V. (2015). OneYear randomized controlled trial and follow-up of integrated neurocognitive therapy for schizophrenia outpatients. Schizophrenia Bulletin, 41(3), 604-616.

Muller, F., Simion, A., Reviriego, E., Galera, C., Mazaux, J., Barat, M., \& Joseph, P. (2010). Exploring theory of mind after severe traumatic brain injury. Cortex, 46(9), 1088-1099.

Neumann, D., Babbage, D.R., Zupan, B., \& Willer, B. (2014). A randomized controlled trial of emotion recognition training after traumatic brain injury. Journal of Head Trauma Rehabilitation, 30(3), 12-23.

Neumann, D., Zupan, B., Babbage, D.R., Radnovich, A.J., Tomita, M., Hammond, F., \& Willer, B. (2012). Affect recognition, empathy, and dysosmia after traumatic brain injury. Archives of Physical Medicine and Rehabilitation, 93(8), 1414-1420.

Nowicki, S., \& Duke, M.P. (1994). Individual differences in the nonverbal communication of affect the Diagnostic Analysis of Nonverbal accuracy scale. Journal of Nonverbal Behavior, 18(1), 9-35.

Ogrodniczuk, J.S., Sochting, I., Piper, W.E., \& Joyce, A.S. (2012). A naturalistic study of alexithymia among psychiatric outpatients treated in an inte- 
grated group therapy program. Psychology and Psychotherapy, 85(3), 278-291.

Paquin, K., Wilson, A.L., Cellard, C., Lecomte, T., Potvin, S. (2014). A systematic review on improving cognition in schizophrenia: Which is the more commonly used type of training, practice or strategy learning? BMC Psychiatry, 14(1), 139-158.

Patterson, T.L., Moscona, S., McKibbin, C.L., Davidson, L., \& Jeste, D.V. (2001). Social skills performance assessment among older patients with schizophrenia. Schizophrenia Research, 48(2), 351-360.

Pawelak, U. (2004). Short versions of "Vienna Emotion Recognition Tasks" (VERT-K) and Vienna Memory of Emotion Recognition Tasks (VIEMER-K). Construction and application. Unpublished master thesis, University of Vienna, Austria.

Penn, D.L., Corrigan, P.W., Bentall, R.P., Racenstein, J.M., \& Newman, L. (1997). Social cognition in schizophrenia. Psychological Bulletin, 121(1), 114132.

Penn, D.L., Mueser, K.T., Doonan, R., \& Nishith, P. (1995). Relations between social skills and ward behavior in chronic schizophrenia. Schizophrenia Research, 16(3), 225-232.

Peyroux, E., \& Franck, N. (2014). RC2S: A cognitive remediation program to improve social cognition in schizophrenia and related disorders. Frontiers in Human Neuroscience, 8, 1-11. doi: 10.3389/2014/00400.

Pino, M.C., Pettinelli, M., Clementi, D., Gianfelice, C., \& Mazza, M. (2015). Improvement in cognitive and affective theory of mind with observation and imitation treatment in subjects with schizophrenia. Clinical Neuropsychiatry, 12(3), 64-72.

Plana, I., Lavoie, M., Battaglia, M., \& Achim, A.M. (2014). A meta-analysis and scoping review of social cognition performance in social phobia, posttraumatic stress disorder and other anxiety disorders. Journal of Anxiety Disorders, 28(2), 169-177.

Radice-Neumann, D., Zupan, B., Tomita, M., \& Willer, B. (2009). Training emotional processing in persons with brain injury. Journal of Head Trauma Rehabilitation, 24(5), 313-323.

Roberts, D.L., Combs, D.R., Willoughby, M., Mintz, J., Gibson, C., Rupp, B., \& Penn, D.L. (2014). A randomized, controlled trial of Social Cognition and Interaction Training (SCIT) for outpatients with schizophrenia spectrum disorders. British Journal of Clinical Psychology, 53(3), 281-298.

Roberts, D. L., \& Penn, D.L. (2009). Social cognition and interaction training (SCIT) for outpatients with schizophrenia: A preliminary study. Psychiatry Research, 166(2), 141-147.

Roberts, D. L., Penn, D. L., \& Combs, D. R. (2016). Social cognition and interaction training (SCIT): Clinician guide. New York: Oxford University Press.

Roberts, D.L., \& Velligan, D.I. (2012). Can social functioning in schizophrenia be improved through targeted social cognitive intervention?
Rehabilitation Research and Practice, 2012, 1-8. doi: 10.1155/2012/742106.

Rocha, N.B.F., \& Queirós, C. (2013). Metacognitive and social cognition training (MSCT) in schizophrenia: A preliminary efficacy study. Schizophrenia $R e$ search, 150(1), 64-68.

Roncone, R., Mazza, M., Frangou, I., De Risio, A., Ussorio, D., Tozzini, C., \& Casacchia, M. (2004). Rehabilitation of theory of mind deficit in schizophrenia: A pilot study of metacognitive strategies in group treatment. Neuropsychological Rehabilitation, 14(4), 421-432.

Rosen, A., Hadzi-Pavlovic, D., \& Parker, G. (1989). The life skills profile: A measure assessing function and disability in schizophrenia. Schizophrenia Bulletin, 15(2), 325-337.

Rosenthal, R. (1979). The file drawer problem and tolerance for null results. Psychological Bulletin, 86, 638-664.

Rosenthal, R., Hall, J.A., DiMatteo, M.R., Rogers, P.L., \& Archer, D. (1979). Sensitivity to nonverbal communication: The PONS Test. Baltimore: Johns Hopkins University Press.

Rowe, A.D., Bullock, P.R., Polkey, C.E., \& Morris, R.G. (2001). "Theory of Mind" impairments and their relationship to executive functioning following frontal lobe excisions. Brain, 124(3), 600-616.

Rus-Calafell, M., Gutiérrez-Maldonado, J., OrtegaBravo, M., Ribas-Sabaté, J., \& Caqueo-Urízar (2013). A brief cognitive-behavioural social skills training for stabilised outpatients with schizophrenia: A preliminary study. Schizophrenia Research, 143(2), 327-336.

Russell, T.A., Green, M.J., Simpson, I., \& Coltheart, M. (2008). Remediation of facial emotion perception in schizophrenia: Concomitant changes in visual attention. Schizophrenia Research, 103(1), 248-256.

Sachs, G., Winklbaur, B., Jagsch, R., Lasser, I., Kryspin-Exner, I., Frommann, N., \& Wölwer, W. (2012). Training of affect recognition (TAR) in schizophrenia- Impact on functional outcome. Schizophrenia Research, 138(2), 262-267.

Samamé, C. (2013). Social cognition throughout the three phases of bipolar disorder: A state-of-the-art overview. Psychiatry Research, 210(3), 1275-1286.

Samur, D., Tops, M., Schlinkert, C., Quirin, M., Cuijpers, P., \& Koole, S.L. (2013). Four decades of research on alexithymia: moving toward clinical applications. Frontiers in Psychology, 4, 1-4. doi: 10.3389/fpsyg.2013.00861.

Snowden, J.S., Gibbons, Z.C., Blackshaw, A., Doubleday, E., Thompson, J., Craufurd, D., Foster, J., \& Neary, D. (2003). Social cognition in frontotemporal dementia and Huntington's disease. $\mathrm{Neu}$ ropsychologia, 41(6), 688-701.

Statucka, M., \& Walder, D.J. (2013). Efficacy of social cognition remediation programs targeting facial affect recognition deficits in schizophrenia: A review and consideration of high-risk samples and sex differences. Psychiatry Research, 206(2), 125-139. 
Subic-Wrana, C., Beutel, M.E., Knebel, A., \& Lane, R. D. (2010). Theory of mind and emotional awareness deficits in patients with somatoform disorders. Psychosomatic Medicine, 72(4), 404-411.

Swart, M., Kortekaas, R., \& Aleman, A. (2009). Dealing with feelings: Characterization of trait alexithymia on emotion regulation strategies and cognitiveemotional processing. PLOS ONE, 4(6), 1-7.

Tas, C., Danaci, A.E., Cubukcuoglu, Z., \& Brüne, M. (2012). Impact of family involvement on social cognition training in clinically stable outpatients with schizophrenia- A randomized pilot study. Psychiatry Research, 195(1), 32-38.

Taylor, G. J., \& Bagby, R.M. (2012). The alexithymia personality dimension. In T.A. Widiger (Ed.). The Oxford handbook of personality disorders (pp. 179), Oxford: Oxford University Press.

Taylor, J.D.A., Taylor, G.J., \& Bagby, R.M. (1993). Alexthymia and the recognition of facial expressions of emotion. Psychotherapy and Psychosomatics, 59(34), 197-202.

Taylor, R., Cella, M., Csipke, E., Heriot-Maitland, C., Gibbs, C., \& Wykes, T. (2015). Tackling social cognition in schizophrenia: A randomized feasibility trial. Behavioural and Cognitive Psychotherapy, 44(3), 306-317.

Tottenham, N. (1998). MacBrain face stimulus set. John, D. and Catherine T. Chicago, IL: MacArthur Foundation Research Network on Early Experience and Brain Development.

Tottenham, N., Tanaka, J.W., Leon, A.C., McCarry, T., Nurse, M., Hare, T.A., ... Nelson, C. (2009). The NimStim set of facial expressions: Judgments form untrained research participants. Psychiatry Research, 168(3), 242-249.

Turner-Brown, L.M., Perry, T.D., Dichter, G.S., Bodfish, J.W., \& Penn, D.L. (2008). Brief report: Feasibility of social cognition and interaction training for adults with high functioning autism. Journal of Autism and Developmental Disorders, 38(9), 1777-1784.

Vanheule, S., Verhaeghe, P., \& Desmet, M. (2011). In search of a framework for the treatment of alexithymia. Psychology and Psychotherapy: Theory, Research and Practice, 84(1), 84-97.
Varnum, M.E.W., Grossman, I., Kitayama, S., \& Nisbett, R.E. (2010). The origin of cultural differences in cognition: Evidence for the social orientation hypothesis. Current Directions in Psychological Science, 19(1), 9-13.

Vauth, R., Rüsch, N., Wirtz, M., \& Corrigan, P.W. (2004). Does social cognition influence the relation between neurocognitive deficits and vocational functioning in schizophrenia? Psychiatry Research, 128(2), 155-165.

Veltro, F., Mazza, M., Vendittelli, N., Alberti, M., Casacchia, M., \& Roncone, R. (2011). A comparison of the effectiveness of problem solving training and of cognitive-emotional rehabilitation on neurocognition, social cognition and social functioning in people with schizophrenia. Clinical Practice \& Epidemiology in Mental Health, 7, 123132

Wallander, J.L., Conger, A.J., \& Conger, J.C. (1985). Development and evaluation of a behaviourally referenced rating system for heterosocial skills. Journal of Behavioral Assessment, 7(2), 137153 .

Whittington, J., \& Holland, T. (2011). Recognition of emotion in facial expression by people with PraderWilli syndrome. Journal of Intellectual Disability Research, 55(1), 75-84.

Wingbermühle, E., Egger, J.I.M., Verhoeven, W.M.A., Van der Burgt, I., \& Kessels, R.P.C. (2012). Affective functioning and social cognition in Noonan syndrome. Psychological Medicine, 42(2), 419-426.

Wölwer, W., \& Frommann, N. (2011). Social-cognitive remediation in schizophrenia: generalization of effects of the training of affect recognition (TAR). Schizophrenia Bulletin, 37(suppl. 2), 63-70.

Wölwer, W., Frommann, N., Halfmann, S., Piaszek, A., Streit, M., \& Gaebel, W. (2005). Remediation of impairments in facial affect recognition in schizophrenia: Efficacy and specificity of a new training program. Schizophrenia Research, 80(2-3), 295-303.

Zupan, B. (2009, April). Emotional Inferencing Stories Task. Poster presented at Department of Applied Linguistic Research Day, St. Catharines, ON: Brock University. 